\title{
Novel Multifunctional Magnetic Nanoparticles $₫ A n$ Efficient Theranostic Platform for Magnetic Resonance Imaging and Targeted Therapy of Cervical Cancer
}

\section{Shu Yao}

Shandong University Qilu Hospital

\section{Li Li}

Shandong University Qilu Hospital

\section{Chang Liu}

Shandong University Qilu Hospital

\section{Ziying Wang}

Shandong University Qilu Hospital

Hong Liu

Shandong University Institute of Crystal Materials

\section{Xuantao Su}

Shandong University School of Microelectronics

\section{Zaijun Lu}

Shandong University School of Chemistry and Chemical Engineering

\section{Zhiping Liu}

Shandong University School of Control Science and Engineering

\section{Xu Qiao}

Shandong University School of Control Science and Engineering

\section{Li Song}

Shandong University Qilu Hospital

\section{Ran Chu}

Shandong University Qilu Hospital

\section{Jinyu Meng}

Shandong University Qilu Hospital

\section{Xiyu Pan}

Shandong University Qilu Hospital

Kun song ( $\nabla$ songkun2001226@sdu.edu.cn )

Shandong University Qilu Hospital https://orcid.org/0000-0001-6110-2770

\section{Beihua Kong}

Shandong University Qilu Hospital 


\section{Research}

Keywords: Iron oxide nanoparticles, Magnetic targeting, Magnetic resonance imaging, Cervical cancer, Magnetic hyperthermia

Posted Date: October 11th, 2021

DOI: https://doi.org/10.21203/rs.3.rs-957553/v1

License: (c) (i) This work is licensed under a Creative Commons Attribution 4.0 International License. Read Full License 


\section{Abstract}

Background: The high incidence and mortality rates of cervical cancer pose a serious threat to women's health. Traditional chemotherapy has inevitable drawbacks of nonspecific tumor targeting, high toxicity, and poor therapeutic efficiency. In order to overcome these shortcomings, a novel multifunctional magnetic nanoparticles drug delivery system with tumor targeting and magnetic resonance imaging was developed to achieve precise diagnosis and targeted tumor killing effects.

Methods: Transmission electron microscopy, dynamic light scatting and ultraviolet methods were used to characterize the nanoparticles in vitro. Cell function tests were performed by scratch, transwell and flow cytometry assays. MTT was used to detect the toxicity of the nanoparticles. The motion trajectory, drug release and uptake studies were carried out in vitro. The in vivo pharmacokinetic and drug distribution studies were verified by high performance liquid chromatography methods. Attenuation of the MRI signal by the nanoparticles and their enhanced antitumor efficiency were examined in vivo in mouse cervical cancer models. Sequencing and proteomics were used to detect the key antitumor molecules of the nanoparticles.

Results: Multifunctional magnetic nanoparticles coated with ferric oxide nanoparticles and doxorubicin hydrochloride (DOX-Fe ${ }_{3} \mathrm{O}_{4}$-PEG-PLA-NPs) was prepared successfully. No toxicity was detected of PEGPLA-NP, however, the tumor killing effect was enhanced under the alternating magnetic field significantly. The drug-release study showed that the cumulative release rates of NP groups were much less than free DOX group, while the drug release rate increased under acidic condition. In addition, DOX- $\mathrm{Fe}_{3} \mathrm{O}_{4}-\mathrm{PEG}-\mathrm{PLA}-$ NPs showed improved internalized into carcinoma cells under magnetic field significantly. In vivo studies demonstrated that the combined therapy under an alternating magnetic field displayed improved therapeutic effect when compared with individual therapies as documented by the delayed tumor growth, inhibition of metastasis, and prolonged survival. The in vitro and in vivo MRI results showed that the multifunctional magnetic nanomaterial had a better MRI signal reduction effect and a higher T2 relaxation rate.

Conclusions: We developed an cervical cancer targeting nano-carrier drug delivery system successfully, which showed perfect excellent T2 contrast magnetic resonance imaging, chemotherapy-sensitizing, tumor targeting, and anti-tumor effect, thus have the potential to be a new theranostic strategy for ovarian cancer patients.

\section{Background}

Cervical cancer is the fourth most common cancer in women, with approximately 570000 new cases and 311000 deaths occurred in 2018; however, the incidence of cervical cancer is particularly high in China, with 106000 new cases and 48000 deaths ${ }^{[1,2]}$. Currently, cervical cancer treatment consists of surgery or chemoradiotherapy for early-stage disease and concurrent chemoradiation for advanced-stage patients. However, these conventional treatment strategies are highly aggressive or nonspecific and often 
accompanied by serious side effects ${ }^{[2]}$. The above traditional treatment regimen has not significantly improved the prognosis of patients or prolonged patient survival ${ }^{[3]}$. Therefore, new treatment strategies are urgently needed for the diagnosis and treatment of cervical cancer.

Doxorubicin (DOX) is an anthracycline glycoside antibiotic originally produced by Streptomyces peucetius var. caesius ${ }^{[4]}$. DOX exerts its cytotoxic effects as a DNA intercalating agent to inhibit DNA and RNA biosynthesis. Thus, DOX is widely used as either a single agent or in combination with other chemotherapeutic regimens to treat various kinds of solid tumors as while as shown substantial treatment potential and is regarded as one of the most potent FDA-approved chemotherapeutic drugs ${ }^{[5]}$. However, its application has been limited by its poor water solubility, high toxicity, and side effects such as cardiotoxicity, hepatotoxicity and the suppression of bone marrow hematopoietic function ${ }^{[6]}$. The high rate of DOX toxicity has prompted the development of alternative treatment strategies to reduce the drug's serious side effects. This includes the use of encapsulated drug delivery by various nanocarriers, the new drug delivery systems and administration routes for DOX to increase its tissue selectivity and reduce its toxicity profile.

Early accurate diagnosis and precise targeted tumor therapy strategies are the key factors to improve the prognosis of patients and significantly prolong the survival time of tumor patients. A large number of articles have reported on the use of nanomaterials to carry chemotherapy drugs for the treatment of cervical cancer and showed excellent tumor killing effect, such as the codelivery of chemotherapeutic agents by nanocarriers ${ }^{[7]}$, gene therapy ${ }^{[8]}$, and recombinant protein therapy. In recent years, advances in nanoscience and nanomedicine have led to the development of multifunctional integrated nanocarriers for molecular and cellular imaging, specific tumor therapy, and cancer detection and screening ${ }^{[9-11]}$. Magnetic nanoparticles (MNPs) have been investigated for decades as drug delivery systems because of their high magnetic responsiveness, biodegradability, biocompatibility ${ }^{[12]}$, high delivery efficiency and potential targeting functions. Iron oxide nanoparticles (IONPS) are considered candidates for biomedical applications since they $(\mathbb{\nabla})$ possess unique magnetic properties derived from their ability to strengthen tissue protons to relax, resulting in enhanced Magnetic resonance imaging (MRI), ( $($ ) With nanomotor characteristics under external magnetic field attraction, it can autonomously move to the target site and increase its tumor tissue penetration ability; $(\mathbb{\nabla})$ Generating magnetic hyperthermia( $\mathrm{MH})$ and reactive oxygen species (ROS) under the alternating magnetic field; and $(\mathbb{\nabla})$ are easily modified to adjust their physicochemical parameters to function at the cellular level. Moreover, iron oxide nanoparticles are the only MNPs approved for clinical use by the US Food and Drug Administration (FDA) ${ }^{[13,14]}$. Iron oxide nanoparticles (IONPS) are considered to be a suitable candidate for biomedical applications because of their molecular transport ${ }^{[15]}$, anemia ${ }^{[16]}$, magnetic targeted therapy ${ }^{[17]}, \mathrm{MRI}^{[18]}$ and hyperthermia ${ }^{[19]}$ properties.

In this study, we successfully constructed a novel PEG-PLA-based polymeric nanocarrier co-encapsulated with DOX and IONPS using a single emulsion evaporation method. The motion and magnetic targeting ability of the material was investigated through its motion track in vitro. The slow-release properties of 
multifunctional magnetic nanoparticles were analyzed by in vitro release experiments, pharmacokinetic experiments and tissue distribution studies. The in vitro uptake and tissue distribution experiments confirmed the magnetic targeting of this multifunctional nanoparticle to tumor cells and tissues. Cell function experiments, toxicity experiments, anti-tumor efficacy studies, and sequencing results showed that the tumor-killing mechanism of multifunctional nanoparticles were that magnetic nanoparticles generate magnetic heat and active oxygen with tumor-killing ability under the action of an alternating magnetic field. The synergistic effects of magnetic heat, reactive oxygen species and chemosensitization promote the apoptosis of tumor cells, and then realize the killing effect of tumor cells. In addition to verifying the sustained release effect, tumor targeting properties and excellent anti-tumor effects of multifunctional magnetic nanoparticles, we also investigated the ability of multifunctional magnetic nanoparticles as contrast agents in MRI imaging. Taken together, this work not only presents evidence showing that $\mathrm{DOX}-\mathrm{Fe}_{3} \mathrm{O}_{4}$-PEG-PLA-NPs is a potentially viable therapeutic paradigm to cancer therapy; It also suggests that IONPS promise in offering emerging therapies to revolutionize nanomedicine in the diagnosis and treatment of a myriad of human diseases.

\section{Results}

\section{Synthesis and Characterization of DOX-Fe $\mathrm{O}_{3}-\mathrm{PEG}-\mathrm{PLA}-\mathrm{NPs}$}

As outlined in Figure $1 \mathrm{a}$, the flow chart of DOX-Fe $\mathrm{O}_{3} \mathrm{O}_{4}$-PEG-PLA-NP, which indicates the successful preparation of PEG-PLA multifunctional nanocarriers containing magnetic $\mathrm{Fe}_{3} \mathrm{O}_{4}$ and $\mathrm{DOX}$ at the core using oil-in-water emulsion and subsequent solvent evaporation methods including two steps: 1) synthesis of amphiphilic polymeric nanocarrier PEG-PLA, and 2) DOX and IONPS are encapsulated into PEG-PLA nanomaterials. As visualize by transmission electron microscopy (TEM), PEG-PLA-NP, DOX-PEGPLA-NP and $\mathrm{Fe}_{3} \mathrm{O}_{4}$-DOX-PEG-PLA-NP exhibited uniform particle size and excellent dispersion. As measured by dynamic light scattering (DLS), the size of PEG-PLA-NP is 50nm, while DOX and $\mathrm{Fe}_{3} \mathrm{O}_{4}$ loaded increase the size of DOX-PEG-PLA-NP and $\mathrm{Fe}_{3} \mathrm{O}_{4}$-DOX-PEG-PLA to $90 \mathrm{~nm}$ and $120 \mathrm{~nm}$ respectively, the particle sizes of the three nanocarriers were similar to those measured by transmission electron microscopy (Figure 1b). As showed the fluorescence spectra of FITC coupled with different nanocarriers. The excitation wavelength is $488 \mathrm{~nm}$, and the emission wavelength ranges from $500 \mathrm{~nm}$ and $750 \mathrm{~nm}$ (Figure 1c). Figure 1d shows the UV absorption spectra of PEG-PLA-FITC empty nanoparticles (blue), PEG-PLA-FITC containing DOX nanoparticles (red), and PEG-PLA-FITC containing $\mathrm{Fe}_{3} \mathrm{O}_{4}$ and DOX nanoparticles (black). Figure 1e shows the infrared spectra of the PEG-PLA-FITC, PEG-PLA-DOX-FITC and $\mathrm{Fe}_{3} \mathrm{O}_{4}$-DOX-PEG-PLA-FITC nanoparticles. MTT assay was used to detect the cytotoxicity of PEG-PLA-NP to cervical cancer cells (Hela) at different concentrations and for different duration. The results showed that the cytotoxicity gradually increased with the increase of the concentration. When the concentration was $100 \mu \mathrm{g} / \mathrm{mL}$ and the action time was $72 \mathrm{~h}$, the survival rate of the tumor was $59.02 \%$. MTT results confirmed the low toxicity of PEG-PLA nanomaterials (Figure 1f). Figure $1 \mathrm{~g}$ shows the magneto-thermal transformation of different $\mathrm{Fe}_{3} \mathrm{O}_{4}$ concentrations $(100-1000 \mu \mathrm{g} / \mathrm{mL})$ under the alternating magnetic field, 
which shows concentration-dependent and time-dependent. When the concentration of $\mathrm{Fe}_{3} \mathrm{O}_{4}$ is 1000 $\mu \mathrm{g} / \mathrm{mL}$, the temperature is $56.3 \pm 0.374^{\circ} \mathrm{C}$, which is much higher than that of $\mathrm{H}_{2} \mathrm{O}\left(30.367 \pm 0.379^{\circ} \mathrm{C}\right)$. Photothermal images of $\mathrm{Fe}_{3} \mathrm{O}_{4}$-DOX-PEG-PLA-FITC nanoparticles and water under the alternating magnetic field (Figure $1 \mathrm{~h}$ ).

\section{Encapsulation rate and drug loading}

The encapsulation rate is defined as the weight percentage of DOX in the PEG-PLA-NPs. The DOX encapsulation and drug loading rates into the DOX-Fe $\mathrm{O}_{3}$-PEG-PLA-FITC micellar nanoparticles were $79.9 \%$ and $23.4 \%$, respectively. The encapsulation and drug loading rates into the DOX-PEG-PLA-FITC nanoparticles were $75.5 \%$ and $21.9 \%$, respectively.

\section{Drug release profile in vitro}

Figure $2 a$ and $2 b$ show the release results of the different drug-loaded nanoparticles in vitro. The in vitro release of DOX-NPs under neutral $(\mathrm{pH} \mathrm{7.4)}$ and acidic $(\mathrm{pH}$ 6.5) conditions presented a continuous release pattern. In general, after the same time and at the same $\mathrm{pH}$, the release efficiency of each of the nanocarrier groups was much lower than that of free DOX group, but there was no significant difference between the two drug-loaded nanomicrogroups, which proved the sustained release effect of the nanocarriers in vitro. The drug release rate of $\mathrm{DOX}-\mathrm{NP}_{\mathrm{S}}$ at $\mathrm{pH} 7.4$ was slower than that at $\mathrm{pH} 6.5$, demonstrating the $\mathrm{pH}$ sensitivity of the nanocarrier. After incubation for $160 \mathrm{~h}$, approximately $35 \%$ of the drugs were released at $\mathrm{pH} 6.5$, while the release rate was $25 \%$ at $\mathrm{pH} 7.4$.

\section{Pharmacokinetics}

The standard curves of DOX in tissue and plasma samples were established, and linear regression analysis obtained a linear regression equation between the DOX concentration $(X)$ and the area under the curve $(Y)$ (Figure S1). The pharmacokinetic characteristics are shown in Figure 2c The elimination periods $\left(\mathrm{T}_{1 / 2}\right)$ of DOX-Fe $\mathrm{O}_{3}$-PEG-PLA and DOX-PEG-PLA were $15.92 \mathrm{~h}$ and $13.47 \mathrm{~h}$, respectively, which were significantly longer than that of the free DOX group $(6.41 \mathrm{~h}, p<0.05)$. The AUCs of DOX-Fe $\mathrm{O}_{4}-\mathrm{PEG}-\mathrm{PLA}$ and DOX-PEG-PLA were $159.81(\mu \mathrm{g} / \mathrm{mL} / \mathrm{h})$ and $132.72(\mu \mathrm{g} / \mathrm{mL} / \mathrm{h})$, respectively $(p>0.05)$, which were approximately 3 times that of free DOX group $(\mu \mathrm{g} / \mathrm{mL} / \mathrm{h})(p<0.05)$. The elimination period $\left(T_{1 / 2}\right)$ in the nanocarrier groups were significantly greater than that of the free drug group, and the AUC values were much greater than that of the free DOX group. Based on these results, given the same amount of drug, the drugs in the nanocarrier groups can circulate in the body and act for a longer time, thus having a stronger killing effect on tumors. In addition to the results of the drug release studies, the pharmacokinetics also confirmed the slow release effects of the nanocarriers.

\section{Cellular cytotoxicity of the nanocarriers in vitro}

Figure 3 shows the toxic effects of different drug-loaded nanoparticles on HeLa cells. Figure $3 a$ show the effects of free DOX, DOX-PEG-PLA-NP and DOX-Fe $\mathrm{F}_{3} \mathrm{O}_{4}$-PEG-PLA-NP on tumor cell toxicity with or without alternating magnetic field, respectively. The results showed that the toxicity to the cells was positively 
correlated with drug concentration and time of action. After treatment with the same DOX concentration for the same time with the same external variable magnetic field, the two drug-loaded nanocarrier groups were more toxic to HeLa cells than the free DOX group $(p<0.05)$. Under the action of an external magnetic field, the DOX-Fe $\mathrm{O}_{3} \mathrm{O}_{4}$-PEG-PLA-NP group had the most obvious tumor killing effects. When the DOX dose was $4 \mu \mathrm{g} / \mathrm{mL}$ (72 h), the cell inhibition rates of the DOX, DOX-DOX-PEG-PLA-NP and DOX-Fe $\mathrm{O}_{3} \mathrm{O}_{4}$-PEG-PLANP groups without alternating magnetic field were $69.30 \%, 54.80 \%, 58.04 \%$, respectively $(p<0.05)$, while under AMF the cell inhibition rates of the three groups were $77.38 \%, 80.18 \%, 88.04 \%$, respectively. The above results all prove that the alternating magnetic field improves the tumor cell killing effects of the magnetic nanocarrier. Interestingly, the killing ability of the free DOX group increased rapidly after $8 \mathrm{~h}$ of treatment, while the killing ability of the two nanocarriers increased rapidly after $48 \mathrm{~h}$ of incubation. This further proved the sustained release effect of the drug-loaded nanocarriers. Figure $3 b$ shows the ability of different groups to produce reactive oxygen species (ROS) with or without alternating magnetic field. Blue fluorescence represents DAPI staining nucleus, and green fluorescence represents ROS content.

\section{In vitro functional test}

Figure $4 \mathrm{a}$ and $4 \mathrm{~d}$ display the results of the scratch experiment. The results showed that after $24 \mathrm{~h}$ of treatment, the migration rates of the tumor cells in the four nanoparticle groups were significantly reduced compared with the blank control group $(p<0.05)$, and the DOX-Fe $\mathrm{O}_{4}$-PEG-PLA-NP(AMF) group showed the weakest tumor cell migration ability. Figure $4 \mathrm{~b}$ and $4 \mathrm{e}$ shows the results of flow cytometry. The effects of each group on tumor cell apoptosis were analyzed by annexin V-FITC and PI. The results showed that the probability of tumor cell apoptosis in the nanocarrier group and the free DOX group significantly improved compared to the blank control group $(p<0.05)$. The apoptosis rate in the DOX$\mathrm{Fe}_{3} \mathrm{O}_{4}$-PEG-PLA-NP(AMF) group was $51.36 \%$, while the apoptotic rates of the other four groups were $7.07 \%, 36.33 \%, 33.09 \%$ and $36.37 \%$, respectively. Figure $4 \mathrm{c}$ and $4 \mathrm{f}$ shows the transwell assays were used to detect the influence of the different treatments on the invasion ability of tumor cells. Both scratch test and Tranwell test confirmed DOX-Fe $\mathrm{O}_{3} \mathrm{O}_{4}$-PEG-PLA-NP group under the alternating magnetic field could reduced the invasion and migration ability of tumors significantly. The functional experiment results in Figure 4 demonstrated that the alternating magnetic field promoted the apoptosis of tumor cells , as while as reduced the metastasis and invasion ability of tumor cells for the magnetic nanocarriers.

\section{Cellular uptake results in vitro}

The results of the cellular uptake experiment are shown in Figure 5. DAPI was used to stain the nucleus to detect the cell density of each group. The results showed that there was no statistically significant difference between the groups $(p>0.05)$, indicating the same number of cells in each group. Figure $5 b-c$ show the uptake fluorescence intensities of the tumor cells in the different drug groups. The free DOX drug group reached peak uptake at 2-4 h, while the DOX-PEG-PLA-NP group reached peak uptake at $2 \mathrm{~h}$. Under the magnetic field, the DOX-Fe $\mathrm{O}_{3}$-PEG-PLA-NP group uptake reached its peak at $1 \mathrm{~h}$, proving that the external magnetic field can promote the rapid uptake of the nanocarriers by the tumor cells. In addition, the $\mathrm{DOX}-\mathrm{Fe}_{3} \mathrm{O}_{4}$-PEG-PLA-NP group was taken up by tumor cells to a greater extent under the 
external magnetic field, which further proved the magnetic targeting characteristics of DOX- $\mathrm{Fe}_{3} \mathrm{O}_{4}$-PEGPLA-NP in vitro. For example, after incubation for $1 \mathrm{~h}$, the fluorescence intensities of HeLa cells in the different groups (DOX, DOX-PEG-PLA-NP, DOX- $\mathrm{Fe}_{3} \mathrm{O}_{4}$-PEG-PLA-NP and DOX-Fe $\mathrm{O}_{4}$-PEG-PLA-NP (magnetic field)) were $3.875 \mathrm{au}, 12.908 \mathrm{au}, 7.056 \mathrm{au}$ and $27.216 \mathrm{au}(p<0.05)$, respectively. The uptake of PEG-PLA nanoparticles reached a peak at $4 \mathrm{~h}$, but its cell uptake capacity did not change significantly with increasing time, proving that cells can continue to take up nanoparticles smoothly. Figures $5 \mathrm{~d}$-f show the mean square displacement diagrams and plane trajectories of nanoparticles under the attraction of an external magnet. The results show that magnetic nanomaterials can be targeted to move to a local area under the attraction of an external magnet, and the movement speed is faster.

\section{Organizational distribution of DOX in the different groups}

The levels of DOX in the plasma and other tissues at different time points after drug delivery are shown in Figure 6 . First, compared with the free DOX group $(1 \mathrm{~h})$, the peak arrival times of the two nanocarrier groups (12 h) were extended in all tissues, while under the effect of external magnetic field, the peak arrival times of DOX-Fe $\mathrm{O}_{4}$-PEG-PLA-NP was $2 \mathrm{~h}$, as shown in Figure 6 . These results once again confirmed the sustained release effects of the nanocarriers observed from the results of the pharmacokinetic studies. Second, as shown in Figure $6 \mathrm{~b}, 2 \mathrm{~h}$ after administration under the action of an external magnetic field, the DOX concentration in tumor tissues $(190.4003 \pm 0.800207 \mu \mathrm{g} / \mathrm{mL})$ of DOX$\mathrm{Fe}_{3} \mathrm{O}_{4}$-PEG-PLA-NP (magnet) group was much higher than that in the free DOX $(68.3168 \pm 0.078 \mu \mathrm{g} / \mathrm{mL}, p$ $<0.005)$, DOX-PEG-PLA-NP $(59.6536 \pm 0.2673 \mu \mathrm{g} / \mathrm{mL}, p<0.005)$ and DOX-Fe ${ }_{3} \mathrm{O}_{4}$-PEG-PLA-NP $(117.4146$ $\pm 0.093 \mu \mathrm{g} / \mathrm{mL}, p<0.005$ ). All of these results indicate the tumor targeting characteristics of $\mathrm{DOX}-\mathrm{Fe}_{3} \mathrm{O}_{4}-$ PEG-PLA-NPs based on ferroferric oxide magnetic targeting. Third, in the kidneys and spleen (Figure 6e and 6f), the tissue content of DOX in the free DOX group was higher than that of the nanocarrier group at all time points. Free DOX is more likely to accumulate in the reticuloendothelial system, increasing spleen and kidney toxicity. Interestingly, at different time points, the content of DOX in the blood of the free DOX group was lower than that of the nanocarrier group. The nanocarrier group had more drug circulating in the blood and a more durable tumor-killing effect. Figure 6j shows the Prussia blue staining in tumor from mice with the DOX-Fe $\mathrm{O}_{3} \mathrm{O}_{4}$-PEG-PLA-NP group treatments.

\section{Antitumor effects of the different groups in vivo}

The schematic diagram of treatment plans in subcutaneous tumor-bearing mouse models with different treatments (Figure 7a). Image taken with IR thermal camera of Hela tumor-bearing mice with different treatments under AMF (Figure 7b), and Figure 7c shows the tumor temperature curves of tumor region under AMF. Figure 7d shows images of the tumor-bearing mice after 26 days of treatment, as well as the tumor map of the mice. The results show that the volume signal from tumor-bearing mice in the DOX$\mathrm{Fe}_{3} \mathrm{O}_{4}$-PEG-PLA-NP group treated with the alternating magnetic field is relatively weak, which proves that DOX-Fe $\mathrm{O}_{3} \mathrm{O}_{4}$-PEG-PLA-NP has a strong tumor killing effect under the alternating magnetic field. Figure $7 f$ shows the changes in animal weight after different treatments. There was no statistically significant difference between the 5 groups ( $p>0.05$ ), indicating that the nanocarrier had no significant side effects. 
Figure $7 \mathrm{~g}$ shows the tumor growth curve, and the results showed compared with the control group, all treatment groups showed significant antitumor effects $(p<0.05)$. The final tumor size of DOX-Fe $\mathrm{O}_{4}-\mathrm{PEG}-$ PLA-NP(AMF) group was $299.09 \pm 3.76 \mathrm{~mm}^{3}$, which was much smaller than that of DOX-Fe $\mathrm{O}_{4}$-PEG-PLA$\mathrm{NP}\left(325.07 \pm 7.09 \mathrm{~mm}^{3}, p=0.01\right)$, DOX-PEG-PLA-NP group $\left(421.78 \pm 10.01 \mathrm{~mm}^{3}, p<0.05\right)$, free DOX group $\left(577.22 \pm 11.99 \mathrm{~mm}^{3}, p<0.05\right)$ and control group $\left(748.12 \pm 22.58 \mathrm{~mm}^{3}, p<0.05\right)$. The tumor volume-based tumor inhibition rate in the DOX-Fe $\mathrm{O}_{3}$-PEG-PLA-NP(AMF) group was $60.02 \%$, which was approximately 1.37 times and 2.63 times higher than that of the DOX-PEG-PLA-NP group (43.62\%) and free DOX group (22.84\%). Under the alternating magnetic field, DOX-Fe $\mathrm{O}_{3} \mathrm{O}_{4}$-PEG-PLA-NP group showed more excellent tumor killing effect. Tumor weight diagram of tumors with indicated treatments (Figure 7h). Diagram of treatment plans in abdominal tumor-bearing mouse models with different treatments (Figure 8a). Heat map of tumor metastasis frequency in tissues and organs, which show that the invasion ability of DOX-FE304-PEG-PLA-NP (AMF) group to other tissues was the weakest (Figure 8b). Figure $8 \mathrm{c}$ show the Kaplan-Meier survival graph of abdominal tumor-bearing mice after treatment, the median survival time of the five treatment groups were $54,96,64,76$ and 120 days. H\&E analysis of different tissues/organs with different treatments, there was no significant difference in H\&E staining with different groups (Figure 8d). Staining of tumor tissue with different treatments. Ki67 and TUNEL immunohistochemical staining of the tumors was performed to study the antiproliferative activity of the nanoparticles (Figure 8e). The DOX-Fe ${ }_{3} \mathrm{O}_{4}$-PEG-PLA-NP(AMF) treatment showed the best antiproliferative activity, with only $37 \%$ of Ki67+ cells. These results confirm that IV delivery of DOX-Fe $\mathrm{O}_{4}$-PEG-PLA-NP under the AMF conditions to inhibit primary tumor growth through a cooperative antiproliferative effect.

\section{Sequencing}

Figure 9 shows the sequencing results of the tumor tissues in the different groups. The results show that compared with the normal control group, the DOX-Fe $\mathrm{O}_{3}$-PEG-PLA-NP group had 10 significantly differentially expressed RNAs and proteins, and more than half of the differentially expressed proteins were related to apoptosis. Abnormal expression of apoptosis-related molecules was also detected in the other nanocarrier groups. The molecular protein levels once again prove that the antitumor effects of these multifunctional magnetic nanocarriers is caused by apoptosis.

\section{MRI results}

In order to investigate the potential usefulness of the prepared MRI contrast agent, relaxivity measurements were carried out. Samples of two different SPIONs were prepared with different iron concentrations. Figure 10a shows the MRI results of the different materials, and as the iron concentration increased, the $T_{2}$ signal intensity gradually decreased. Figure $10 \mathrm{~b}$ shows the $\mathrm{T} 2$ signal results of different materials under different TE conditions. The concentration-relaxation equations of DOX-Fe $\mathrm{O}_{3}$-PEG-PLA$\mathrm{NP}$, the USPIO standard product and gadopentetate meglumine according to the $1 / \mathrm{T} 2$ value and concentration are $Y=118.39 X-0.8072, Y=24.249 X-0.00807$ and $Y=1.7904 X+0.1962$ when $T E=10$, respectively (Figure 10c). The relaxation rates of the three groups are $118.39 \mathrm{mM}^{-1} \mathrm{~s}^{-1}, 24.249 \mathrm{mM}^{-1} \mathrm{~s}^{-1}$ and $1.7904 \mathrm{mM}^{-1} \mathrm{~s}^{-1}$, respectively. The above results indicate that DOX-Fe $\mathrm{O}_{4}$-PEG-PLA-NP as a nuclear 
magnetic resonance contrast agent has a higher relaxation rate. MRI of mice injected intravenously with different materials at different times (Figure 10d).

\section{Discussion}

In this study, we developed a new active ingredient characterization system, DOX- $\mathrm{Fe}_{3} \mathrm{O}_{4}$-PEG-PLA-NP, which is potentially effective and promising for cancer diagnosis and treatment. Polylactic acid (PLA) is a biodegradable synthetic polymer that has been approved by the US Food and Drug Administration (FDA) for medical applications ${ }^{[20]}$. However, due to its weak hydrophilicity, long degradation time and easy absorption by the liver and kidney, the applications of PLA are limited. In order to overcome these shortcomings, we coupled PEG with PLA to reduce absorption by the reticuloendothelial system ${ }^{[21]}$. In addition to the substance itself affecting the uptake by tumor cells, the size of the charge also affects the absorption capacity by tumor cells. Positively charged nanoparticles will be captured by the reticuloendothelial system (RES) and interact with negatively charged serum components, leading to further accumulation in organs such as the liver and kidney and a shortened blood circulation time. In addition, it has been proven that positively charged nanoparticles have a high affinity for negatively charged cell membranes and therefore have high inherent cell characteristics ${ }^{[22-24]}$. The PEG-PLA-NP material itself has a positive charge. In order to meet this challenge, considering that the external environment of tumor cells is more acidic (approximately $\mathrm{pH}$ 6.5) than the acidity of blood ( $\mathrm{pH} \mathrm{7.4),} \mathrm{a}$ novel PEG-PLA nanocarrier was designed. The carrier was designed to be ideal to stimulate system responsiveness, where these smart polymers could maintain a negative charge in blood circulation and minimize nonspecific adsorption. After accumulation in a weakly acidic environment, they can spontaneously undergo a transformation from a negative to a positive charge, which promotes cell absorption or escape from lysosomes. Today, the use of magnetite nanoparticles has become a useful medical method for applications such as targeted drug delivery, MRI contrast agents ${ }^{[11]}$, magnetic hyperthermia ${ }^{[19]}$, cell separation, and DNA testing. In this study, we encapsulated $\mathrm{Fe}_{3} \mathrm{O}_{4}$ and the tumorkilling chemical drug DOX into PEG-PLA nanocarriers, finally developing DOX-Fe $\mathrm{O}_{3} \mathrm{O}_{4}$-PEG-PLA-NPs with tumor magnetic targeting, tumor imaging and tumor killing, which can be used as targeted macromolecules, imaging tags and a targeted drug delivery system.

As drug and magnetic material carriers, PEG-PLA nanomaterials display three properties: the ability to increase DOX solubility, a slow release effect and the active targeting to tumor tissues. DOX as a chemotherapy drug has two proposed mechanisms in cancer cells: 1) intercalation into DNA and disruption of topoisomerase-II-mediated DNA repair, and 2) production of free radicals, which disturbs the cellular membrane, DNA and proteins ${ }^{[25]}$. DOX is currently in clinical use in the form of doxorubicin hydrochloride because the original drug is insoluble in water, but its lack of tumor targeting limits its clinical applications ${ }^{[6,25]}$. PEG-PLA wraps DOX into nanoparticles, completely solving the problem of DOX solubility, which is one of the advantages of our PEG-PLA-NP drug delivery system. Another advantage of our nanocarrier system is its perfect slow release effects. The results of the drug release, pharmacokinetic, and drug distribution studies showed that the duration of DOX-Fe $\mathrm{O}_{3} \mathrm{O}_{4}$-PEG-PLA-NPs in 
the body circulation ( $T_{1 / 2}$ and peak arrival time) is much longer than that of the free DOX solution. In other words, at the same drug dose, nanocarriers can cause DOX to be more effective against tumors. The reasons affecting the slow release effects of the nanocarrier are as follows. 1) Tumor tissues proliferate rapidly, but the corresponding vascular dysplasia leads to the local acidic environment of the tumor ${ }^{[8,26]}$. The magnetic nanocarrier designed by our research group cleaves PEG-PLA in the acidic environment, thus releasing more drug. 2) The drug is encapsulated in the PEG-PLA nanocarrier, and the circulation of PEG-PLA in the blood weakens absorption by the reticuloendothelial system, so the drug showed reduced metabolism by the liver and kidney. A large amount of drug can therefore circulate in the body for a long time, allowing the drug to be slowly released into tumor tissues. The most important advantage of our nanodrug delivery system is its active targeting of tumor tissues. Fast-growing tumors require new blood vessels (neovascularization) or rerouting of the existing vessels adjacent to the tumors to provide enough oxygen and nutrition for their survival. This generates abnormal fenestrated endothelial structures around the tumors that are highly permeable for IONPs. These leaky vessels, which lack any associated lymphatic drainage, drive a unique process known as the enhanced permeability and retention (EPR) effect ${ }^{[27]}$. Therefore, the EPR effect may be one of the reasons that an increase in the distribution in tumor tissues and an increase tumor killing was observed. In addition, there are many factors that affect the distribution of nanocarriers in the body, such as the target organ type, the size of the nanocarrier, and the surface charge of the nanocarrier ${ }^{[10,28]}$.

This research group designed PEG-PLA nanomaterials coated with IONPs, which have the following advantages. 1) Under the alternating magnetic field (AMF), compared with the free DOX and DOX-PEGPLA-NP groups, HeLa cells take more $\mathrm{DOX}-\mathrm{Fe}_{3} \mathrm{O}_{4}-\mathrm{PEG}-\mathrm{PLA}-\mathrm{NP}$ up in vitro. Research on drug distribution in tumor-bearing animals showed that the DOX concentration in tumor tissues of DOX-Fe $\mathrm{O}_{3} \mathrm{O}_{4}$-PEG-PLA-NP group was 2.79-fold greater than that in the free DOX group and 3.19-fold higher than that in the DOXPEG-PLA-NP group after $2 \mathrm{~h}$ tail intravenous injection. Magnetic nanomaterials are driven to target positions using magnetic field gradients. A recent study showed that using an external magnet around tumor sites significantly enhances the targeting ability of peptide-loaded IONPs and decreases liver uptake ${ }^{[29]}$. In this study, more magnetic nanocarriers brought chemotherapeutic drugs into the tumor tissue, which also demonstrated the tumor-targeting properties of the magnetic nanoparticles. In addition to the passive targeting of nanomaterial EPR, magnetic targeting may be another reason for the observed increase in drug distribution in tumor tissues. 2) Iron oxide generates reactive oxygen under the action of AMF, and the mechanism of this ROS killing tumor cells is very complex. It has been identified that various ROS forms in aqueous solution when magnetic nanoparticles are excited by alternating magnetic field, such as hydroxyl radicals $(\cdot \mathrm{OH})$, hydrogen peroxide $\left(\mathrm{H}_{2} \mathrm{O}_{2}\right)$, superoxide radicals $\left(\cdot \mathrm{O}_{2}{ }^{-}\right)$, and singlet oxygen $\left({ }^{1} \mathrm{O}_{2}\right)$. ROS acts on tumor cells and eventually leads to cell death by promoting apoptosis ${ }^{[11]}$. 3 ) Finally, under the action of AMF, the magnetic heat generated by the IONPs kills the tumor ${ }^{[30]}$. Hyperthermia, by definition, is the application of heat above $40^{\circ} \mathrm{C}$ to kill tumor cells, which has been used alone and in combination with chemotherapy and radiation therapy. Higher temperatures (above $42^{\circ} \mathrm{C}$ ) cause necrosis of living cells, denature enzymes, promote functional changes to DNA and RNA, and 
rupture cellular membranes, which releases cellular content, ultimately leading to cell injury and death ${ }^{[31]}$. In addition, increased heat increases the tumor killing effect of chemotherapeutic drugs.

The early diagnosis and treatment of cancer are key factors for good prognosis. Cancer diagnosis using nanotechnology is an emerging field. Substantial efforts have been made in biomedical research to improve the sensitivity and accuracy of early detection methods to diagnose cancer and increase the effectiveness of treatment methods. Currently, the understanding of suitable biomarkers for imaging, the selection of imaging targets and contrast-enhancing materials, and the chemicals required for assembly of bioactive imaging probes is limited. In addition, there are many obstacles in the development of cancer-specific imaging agents, such as 1 ) the delivery of probes to target tissues/tumors; 2 ) biocompatibility and toxicity; 3) stability of the probes and enhancement of effective signals in vivo; and 4) adequate imaging methods and strategies ${ }^{[17,18,32,33]}$. Nanoparticle drug delivery can provide more effective and less harmful solutions to overcome these problems. $\mathrm{Fe}_{3} \mathrm{O}_{4}$ can enhance the sensitivity of MRI imaging. According to the quantum mechanical outer sphere theory, the T2 relativity or spin-spin relaxation is highly dependent on the saturation magnetization of the NPs. The greater T2 relativity is because larger NPs with higher saturation magnetization can afford more effective magnetic relaxations to the protons of water around the NPs ${ }^{[11,34]}$. The contrast effects of NPs are highly size-dependent. By controlling the size, we can achieve a much higher contrast enhancement. The particle size of the nanocarrier designed by our research group is above 100 nanometers, which can not only avoid oversized renal metabolism but also improve the imaging ability of ferric oxide. We used these four advantages of iron trioxide to improve the tumor killing and MRI imaging effects. The function of SPIONs in MRI contrast enhancement is attributed to the ability of SPIONs to change the nuclear spin relaxation of water protons and cause the target area to darken ${ }^{[35]}$. The advantages of magnetic resonance nanotechnology in measuring nanoparticle uptake come from the fact that the data collected are quantitative, have very effective soft tissue characterization and high tissue resolution, and have a tomography modality, which penetrates into the tissue without restriction. Therefore, as an effective MRI contrast agent, iron oxide nanoparticles have great potential in both imaging and image guidance. Specifically, iron oxide nanoparticles can be detected with high sensitivity. In contrast to $\mathrm{Gd}$, iron and polymer components have the advantages of biocompatibility, degradability and low toxicity. The degraded iron will be stored for further biological needs. Iron oxide nanoparticles are converted into elemental iron and finally incorporated into our body reserves or used to form hemoglobin ${ }^{[36]}$.

The excellent sustained-release effects and tumor-targeting properties of the DOX-Fe $\mathrm{O}_{3}$-PEG-PLA-NPs significantly increase the content of $\mathrm{DOX}$ and $\mathrm{Fe}_{3} \mathrm{O}_{4}$ in tumor tissues, which is a prerequisite for improving the killing effects. The research group designed a novel PEG-PLA nanocarrier that uses the local acidic environment of tumor tissue to increase the release of the drugs and magnetic substances. In addition, nanocarriers have an EPR effect, and more nanocarriers are retained in the tumor tissue. Finally, under the action of an external magnetic field, more nanocarriers are attracted to the tumor tissue. The synergy of passive targeting and magnetic targeting have jointly increased the accumulation of drugs and iron for tumor targeting. 
The killing of tumors depends on not only the chemical killing effects of the chemotherapeutic drug but also the action of an external variable magnetic field. Ferric oxide produces magnetic heat locally, which also has a killing effect on tumor cells. In addition, under the $\mathrm{AMF}, \mathrm{Fe}_{3} \mathrm{O}_{4}$ can produce reactive oxygen that can kill tumor cells. These three synergistic killing effects promote the apoptosis of tumor cells and jointly improve the tumor killing effects. If there is a lack of tumor targeting, chemotherapy, magnetic heating and reactive oxygen can kill tumor cells while also killing normal tissues. The research team used tumor targeting properties to focus on multiple tumor-killing mechanisms in tumor tissues. Targeting tumors is equivalent to using the correct commander, and chemotherapy drugs and magnetic substances are equivalent to the combat soldiers. Only precise targeting and effective killing are meaningful for cancer patients.

\section{Conclusion}

In summary, we developed a diagnostic therapy drug delivery system based on chemotherapeutic DOX and magnetic ferric oxide, which promotes targeted tumor delivery and enhances antitumor efficacy. The main research contents include (1) preparation and characterization of the nanomaterials; (2) verification of the sustained-release effects in vivo and in vitro; (3) verification of the passive targeting and magnetic targeting characteristics in vitro and in vivo; (4) validation of drug resistance and antitumor efficacy in vitro and in vivo; and (5) enhanced validation of MRI imaging. In summary, the magnetic nanomaterials successfully prepared by the research group could generate magnetic heat and active oxygen under the action of an alternating magnetic field, and chemotherapy enhance tumor killing. these three killing effects synergistic promote tumor cell apoptosis. The PEG-PLA drug-loaded nanosystem significantly improves the hydrophilicity of chemotherapeutic drugs. As a drug delivery container, the system has shown tumor targeting capabilities (passive and magnetic targeting). Importantly, DOX- $\mathrm{Fe}_{3} \mathrm{O}_{4}$-PEG-PLANPs show excellent antitumor effects and MRI sensitization effect. The further integration of the multiple functions of these nanoparticles in MRI and treatment shows unique advantages (so-called therapeutic therapies). In general, the DOX-Fe $\mathrm{O}_{4}$-PEG-PLA-NP multifunctional magnetic nanosystem (tumor targeted drug delivery systems) based on ferroferric oxide and chemotherapeutic drugs can provide potential development strategies for the diagnosis and treatment of cervical cancer.

\section{Materials And Methods}

(4,5-Dimethylthiazol-2-yl)-2,5-diphenyltetrazolium bromide (MTT) was obtained from Amresco (Solon, OH, USA); potassium bis(trimethylsilyl)amide (KHMDS), dimethyl maleic anhydride, ethylene oxide and lactide were obtained from Xi'an Ruixi Biological Technology Co., Ltd.; D-tocopherol polyethylene glycol 1000 succinate (TPGS) was purchased from Sigma; chloroform was purchased from Sinopharm Chemical Reagent Co., Ltd. (Shanghai, China); and $\mathrm{FeCl}_{3}-6 \mathrm{H}_{2} \mathrm{O}$, ethylene glycol, dimethyl sulfoxide (DMSO), urea, ethylene Diamine Tetraacetic Acid (EDTA), doxorubicin (DOX) and anhydrous ethanol were purchased from Hao Sai Biotechnology Co., Ltd. (Jinan, China). 1640 medium, penicillin-streptomycin, fetal bovine serum (FBS) and $0.25 \%$ trypsin-ethylene diaminetetraacetic acid solution were purchased from Invitrogen- 
Gibco (Waltham, MA, USA). Reactive Oxygen Species Assay Kit was purchased from Hao Sai Biotechnology Co., Ltd. (Jinan, China).

\section{Cell culture}

Human cervical carcinoma (HeLa) cells were purchased from the Shanghai Institute for Biological Sciences (Shanghai, People's Republic of China) and grown in 1640 medium containing $10 \%(\mathrm{v} / \mathrm{v})$ fetal bovine serum (FBS) and 1\% (v/v) penicillin-streptomycin $(100 \mathrm{mg} / \mathrm{mL}$ penicillin $\mathrm{G}$ and $100 \mathrm{mg} / \mathrm{mL}$ streptomycin) at $37^{\circ} \mathrm{C}$ in a $5 \% \mathrm{CO}_{2}$ atmosphere at $95 \%$ relative humidity.

\section{Animals}

Female Sprague-Dawley (6-8 weeks old, $150 \mathrm{~g}$ ) and female nude mice (6-8 weeks old, $20 \mathrm{~g}$ ) were purchased from Weitong Lihua Biotechnology Co., Ltd. (Beijing, China). All animal procedures were performed in accordance with the protocol approved by the Animal Care and Use Committee of Shandong University, Qilu Hospital (DWLL-2019-005).

\section{Preparation of DOX-Fe $\mathrm{O}_{3}$-PEG-PLA-NPs}

\section{Synthesis of oily $\mathrm{Fe}_{3} \mathrm{O}_{4}$}

The synthesis of MNPS-EDTA by the solvent thermal method was prepared by the hydrothermal method using $\mathrm{FeCl}_{3}-6 \mathrm{H}_{2} \mathrm{O}$ as the single Fe ion source. $\mathrm{FeCl}_{3}-6 \mathrm{H}_{2} \mathrm{O}(0.5 \mathrm{~g})$ was dissolved in ethylene glycol $(30 \mathrm{~mL})$ to form a clear solution. Then, urea $(1.8 \mathrm{~g})$ and EDTA $(0.35 \mathrm{~g})$ were added to the mixed solution and vigorously stirred for 30 minutes. The mixture was put into a Teflon-lined stainless steel autoclave (50 $\mathrm{mL}$ ); thereafter, the autoclave was heated to $198^{\circ} \mathrm{C}$ for $6 \mathrm{~h}$ in a furnace and allowed to cool to room temperature. The reaction products were collected with magnets and cleaned and reused in anhydrous ethanol and deionized water. Finally, the nanoparticles were dried in a vacuum oven at $50^{\circ} \mathrm{C}$ for $24 \mathrm{~h}$. On this basis, $\mathrm{Fe}_{3} \mathrm{O}_{4}$ magnetic nanoparticles (MNPs) were prepared without EDTA.

\section{Synthesis of $\mathrm{H}_{2} \mathrm{~N}$-PEG-b-PLA nanoparticle micelles}

With potassium bis(trimethylsilyl)amide (KHMDS) as the initiator, ethylene oxide and lactide were successively added to carry out anionic ring-opening polymerization, and the protecting group was removed by acid hydrolysis to produce the $\mathrm{H}_{2} \mathrm{~N}-\mathrm{PEG}-\mathrm{b}-\mathrm{PLA}$ block copolymer. Then, a certain proportion of dimethyl maleic anhydride was added to obtain HOOC-PEG-PLA.

\section{Synthesis of DOX-PEG-PLA micelles}

First, $500 \mu \mathrm{L}$ of $\mathrm{PEG}_{5 \mathrm{k}}-\mathrm{PLA} \mathrm{A}_{5 \mathrm{k}}(100 \mathrm{mg} / \mathrm{mL}), 1 \mathrm{~mL}$ of $\mathrm{DOX}(2.5 \mathrm{mg} / \mathrm{mL})$ and $2.2 \mathrm{~mL}$ of chloroform were uniformly mixed and added into a mixture of $12 \mathrm{~mL}$ of $1 \%$ PVA and TPGS (external aqueous phase, PVA:TPGS 1:5). An oil-water emulsion was formed by using probe ultrasound for 5 minutes $(80 \mathrm{~W})$ in an ice bath. Finally, the mixture was added to $60 \mathrm{~mL}$ of $0.3 \%$ PVA (dispersed phase) and stirred overnight to 
volatilize the chloroform and solidify the PLA ball surface. A $100 \mathrm{kD}$ ultrafiltration tube was used for ultrafiltration concentration cleaning. Finally, the sample was collected and stored at $4^{\circ} \mathrm{C}$ after a constant volume of pure water was added for a final volume of $10 \mathrm{~mL}$.

\section{Preparation of DOX-Fe ${ }_{3} \mathrm{O}_{4}$-PEG-PLA micelles}

First, $500 \mu \mathrm{L}$ of $\mathrm{PEG}_{5 \mathrm{k}}-\mathrm{PLA}_{5 \mathrm{k}}(100 \mathrm{mg} / \mathrm{mL}), 1 \mathrm{~mL}$ of $\mathrm{DOX}(2.5 \mathrm{mg} / \mathrm{mL})$ and $2.2 \mathrm{~mL}$ of chloroform were uniformly mixed and added into a mixture of $12 \mathrm{~mL}$ of $1 \%$ PVA and TPGS (external aqueous phase, PVA:TPGS 1:5). An oil-water emulsion was formed by using probe ultrasound for 5 minutes $(80 \mathrm{~W})$ in an ice bath. Finally, the mixture was added to $60 \mathrm{~mL}$ of $0.3 \%$ PVA (dispersed phase) and stirred overnight to volatilize the chloroform and solidify the PLA ball surface. A $100 \mathrm{kD}$ ultrafiltration tube was used for ultrafiltration concentration cleaning. Finally, the sample was collected and stored at $4^{\circ} \mathrm{C}$ after a constant volume of pure water was added for a final volume of $10 \mathrm{~mL}$.

\section{Characterization of the nanoparticles}

TEM images were taken with a JEM-2100 HR transmission electron microscope (JEOL, Tokyo, Japan) with a tungsten filament at an accelerating voltage of $200 \mathrm{kV}$. The UV-vis absorption values of the different nanoparticle samples were measured with a UV-vis-NIR spectrophotometer (UV-1780, Shimadzu, Japan). The hydrodynamic size and surface potential of the nanoparticles were determined in the aqueous phase by using a Malvern Zetasizer Nano-ZS (Malvern Instruments, Worcestershire, UK). Fluorescence spectra of the drug-loaded PLA-PEG-FITC nanoparticles were measured with a Tecan multifunctional enzyme standard instrument (excitation wavelength was $488 \mathrm{~nm}$, emission wavelength was $500 \mathrm{~nm}-750 \mathrm{~nm}$ ). Infrared spectra of the nanoparticles were obtained with an FT-IR spectrometer (Nexus 870 FT-IR, Thermo Nicolet, USA). The magneto-thermal conversion efficiency of materials was measured by infrared thermal camera in vitro.

\section{Drug loading and encapsulation rate}

One hundred microliters of DOX-Fe $\mathrm{O}_{3} \mathrm{O}_{4}$-PEG-PLA nanoparticles (a), DOX-PEG-PLA nanoparticles (b), and PEG-PLA nanoparticles (c) were dried in a drying centrifuge tube, and the concentration of nanoparticles was obtained by the weight difference method. Then, $1 \mathrm{~mL}$ of methanol solution was added with ultrasound to dissolve the drug. The content of DOX loaded into the PEG-PLA-FITC micelles was determined by a UV-vis method. PEG-PLA-FITC micella were used as the control group because free FITC could interfere with the measurement. The absorption of DOX at $530 \mathrm{~nm}$ was measured. Equations (1) and (2) were used to calculate the encapsulation rate and drug loading efficiency (DL\%) of the nanoparticles.

\section{Encapsulation rate $=\mathrm{W}_{\mathrm{A}} / \mathrm{W}_{\mathrm{B}} \times 100 \%$ (1)}

\section{Drug load $=W_{A} / W_{C} \times 100 \%$ (2)}


$\mathrm{W}_{\mathrm{A}}$ : DOX (mg) loaded into the PEG-PLA-FITC nanoparticles; $\mathrm{W}_{\mathrm{B}}$ : total dose of DOX (mg); and $\mathrm{W}_{\mathrm{C}}$ : total mass of the drug-loaded nanoparticles (mg).

\section{Release study of nanocarriers in vitro}

The release characteristics of DOX from nanocarriers were studied by the dialysis method. First, freezedried DOX-NPs and free DOX solutions $(1 \mathrm{mg})$ were dissolved in DMSO $(1 \mathrm{~mL})$, transferred to dialysis bags (Snakeskin, Pierce Biotechnology, Rockford, IL, USA) (3500 kDa) and immersed in $50 \mathrm{~mL}$ of neutral saline $(\mathrm{pH}=7.4)$ or acidic saline $(\mathrm{pH}=6.5)$. The vials were shaken horizontally in a shaking water bath (100 $\mathrm{rpm}$ ) at $37^{\circ} \mathrm{C}$ for $160 \mathrm{~h}$. At predetermined time intervals, $5 \mathrm{~mL}$ from each saline sample was collected and replaced with an equal amount of fresh saline. Then, the DOX concentration in the physiological saline samples was analyzed by ultraviolet spectrophotometry to determine the cumulative DOX content released.

\section{Cell culture and cytotoxicity measurements}

The MTT method was used to determine the toxicity of the blank nanocarriers and the different DOX-NP groups to HeLa cells. To detect the effects of the blank nanocarriers on HeLa cell cytotoxicity, different concentrations of the nanocarriers $(5,20,50,100 \mu \mathrm{g} / \mathrm{mL})$ were incubated with the cells for different lengths of time $(8,24,48$ and $72 \mathrm{~h})$. Different drug-loaded nanocarriers with or without the alternating magnetic field conditions used this same method to explore the impacts of different drug concentrations $(0.5,1,2,4 \mu \mathrm{g} / \mathrm{mL}$ in doxorubicin concentration) and different times of action on the tumor cell survival rate. The specific experimental procedures were as follows: HeLa cells were seeded in a 96-well plate at a density of $1 \times 10^{4}$ cells per well. The cells were incubated in an incubator overnight until the cells were $80 \%$ confluent, and then the medium was removed. After the groups with different concentrations of nanoparticles and tumor cells were cocultured, $20 \mu \mathrm{L}$ of MTT solution (in phosphate-buffered saline, 5 $\mathrm{mg} / \mathrm{mL}$ ) was added to each well followed by culturing for another $4 \mathrm{~h}$. Then, $150 \mu \mathrm{L}$ of dimethyl sulfoxide (DMSO) was added to dissolve the blue formazan crystals produced by the proliferating cells, the plates were shaken at $37^{\circ} \mathrm{C}$ for 10 minutes, and a victor 3 microplate reader (PerkinElmer, Boston, MA, USA) was used to measure the absorbance at $490 \mathrm{~nm}$.

\section{In vitro functional tests}

\section{Transwell assay}

Cell invasion was analyzed in a transwell chamber $(8 \mu \mathrm{m})$. In brief, $300 \mu \mathrm{L}$ of complete medium was added to the bottom chamber, and then HeLa cells $\left(1 \times 10^{5}\right.$ cells $\left./ \mathrm{mL}\right)$ were seeded into the upper chambers of 24-well Transwell plates. Then, HeLa cells were incubated with DOX, DOX-PEG-PLA or DOX$\mathrm{Fe}_{3} \mathrm{O}_{4}$-PEG-PLA-NP at a DOX concentration equivalent to $2 \mu \mathrm{g} / \mathrm{mL}$ for $24 \mathrm{~h}$. The concentration of free PEG-PLA-NPs was $8 \mu \mathrm{g} / \mathrm{mL}$. After $24 \mathrm{~h}$, the filter was removed from the plate, and the cells remaining on the upper filter were gently wiped. The cells that migrated to the bottom chamber were fixed with methanol and stained with crystal violet for 2 minutes. The average number of migrating cells was determined from six independent views photographed using a microscope. 


\section{Scratch experiment}

First, two horizontal lines were drawn on the back of a 6-well plate. After the cells were digested, a cell suspension was made and plated, and when the confluence was above $90 \%$, the cells were scratched with a $10 \mu \mathrm{L}$ pipette tip on the bottom horizontal line, followed by gently rinsing with PBS 2-3 times, and replacing the medium with serum-free dosing medium for cell stimulation. Cultivation was continued in a $37^{\circ} \mathrm{C}, 5 \% \mathrm{CO}_{2}$ incubator. After $24 \mathrm{~h}$, pictures were taken under a microscope, and the scratch area was calculated.

\section{Flow cytometry}

Flow cytometry was also utilized to examine the effects of the PEG-PLA-NPs on HeLa cell apoptosis. Briefly, HeLa cells were exposed to DOX, DOX-PEG-PLA or DOX- $\mathrm{Fe}_{3} \mathrm{O}_{4}$-PEG-PLA-NPs at an equivalent DOX concentration of $2 \mu \mathrm{g} / \mathrm{mL}$ for $24 \mathrm{~h}$, while the concentration of free the PEG-PLA-NPs was $50 \mu \mathrm{g} / \mathrm{mL}$. The cells were then washed twice with PBS. Next, the cells were collected and stained with annexin V-FITC/PI for 30 minutes in the dark. Finally, the stained cells were examined by flow cytometry, and the data were analyzed with Flow Jo software (Treestar, Ashland, OR, USA).

\section{Cellular uptake of the nanoparticles}

In order to prove the killing effects of the nanoparticles on tumors in vitro, the uptake capacity of DOX and the DOX-Fe $\mathrm{O}_{3} \mathrm{O}_{4}$-PEG-PLA-NP and DOX-PEG-PLA-NP formulations were evaluated in HeLa cells. We used DAPI to normalize the cell densities of the different groups. HeLa cells were cultured for $24 \mathrm{~h}$, and different culture media sample solutions with the same DOX concentration were added. The cells were incubated with tumor cells for different lengths of time $(1,2,4,8 \mathrm{~h})$, washed with cold PBS 3 times to terminate cellular uptake, fixed with $4 \%$ formaldehyde for 30 minutes and finally washed with PBS 3 times for 10 minutes each time. PBS (5\% Triton-X-100) was permeated for 15 minutes, and then the cells were rinsed with PBS 3 times for 10 minutes each time followed by blocking with PBS (10\% NGS) for $1 \mathrm{~h}$. Cells were then incubated with the specific primary antibody at $37^{\circ} \mathrm{C}$ for 30 minutes. The cells were rinsed with PBS $(0.1 \%$ Tween-20) three times for 10 minutes each. Sections were coated with aluminum foil, incubated with secondary antibodies for 30 minutes, and rinsed with PBS 3 times to remove the secondary antibodies. DAPI was added for 15 minutes of incubation followed by rinsing with PBS ( $0.1 \%$ Tween-20) three times for 10 minutes each time. FITC coupled with PEG-PLA-NP was used for green light excitation imaging in the cellular uptake experiment. The cellular uptake characteristics of the different groups were analyzed by fluorescence microscopy (Olympus, Tokyo, Japan).

\section{Trajectory detection}

HeLa cells were seeded in petri dishes at a density of $1 \times 10^{4}$ cells/well. The culture medium was removed after cell adherence, then add DOX-Fe $\mathrm{O}_{3} \mathrm{O}_{4}$-PEG-PLA-NP solution $(10 \mu \mathrm{g} / \mathrm{mL})$. Attracted by a magnet on one side, the movement of the nanoparticles by microscope and plots the mean square displacement and plane trajectory. 


\section{Pharmacokinetic data analysis in rats}

Female Sprague-Dawley rats ( $\mathrm{n}=6 /$ group) were injected with DOX or DOX-NPs ( $5 \mathrm{mg} / \mathrm{kg} \mathrm{DOX}$ concentration) into the tail vein. Blood samples were collected at predetermined time points $(0.17,0.34$, $0.51,0.68,1,2,4,6,12,24,36,48$ and $60 \mathrm{~h}$ ). Mobile phase (200 $\mu \mathrm{L}$ of methanol and $2 \mathrm{~mL}$ of ethyl ether) was mixed with the blood samples collected at different time points $(200 \mu \mathrm{L})$, followed by vortexing for 5 minutes, centrifuging at 10,000 rpm for 10 minutes, and then drying the samples under nitrogen. Finally, the DOX concentration in each sample was analyzed using high-performance liquid (HPLC).

\section{Tissue-distribution study in tumor-bearing mice}

HeLa cells (Luc-transfected cells) $\left(8 \times 10^{6} / 100 \mu \mathrm{L}\right)$ were subcutaneously inoculated into the right upper limbs of female BALB/c nude mice to establish a subcutaneous tumor mouse model to construct a standard tissue curve and to study drug tissue distribution. To construct the standard curve of DOX, blood and tissue samples (liver, spleen, kidney, heart, lung, uterus, small intestine and tumor) from the tumorbearing mice were homogenized with $5 \mathrm{~mL}$ of PBS. Subsequently, $20 \mu \mathrm{L}$ of different quantitative standard DOX solutions $(1.00,5.00,10.00,25.00,50.00,75.00,100.00,150.00$ and $200 \mu \mathrm{g} / \mathrm{mL})$ were added to the homogenates $(200 \mu \mathrm{L})$, followed by HPLC analysis. A linear regression equation was then determined based on the DOX concentration $(X)$ and AUC value $(Y)$ from the HPLC experiment. Subcutaneous tumor-bearing mice were randomly divided into 4 groups (20 mice/group). Each mouse was intravenously injected with DOX or DOX nanocarrier solution (5 $\mathrm{mg} / \mathrm{kg}$ in DOX concentration). At predetermined time points $(1,2,4,12$, and $24 \mathrm{~h}), 28$ mice in each group were euthanized, their plasma and tissue samples were rapidly collected for HPLC analysis and then the DOX concentrations in the samples were calculated based on the linear regression equation mentioned above.

\section{Anti-tumor efficiency}

Intratumoral injection of DOX-Fe ${ }_{3} \mathrm{O}_{4}$-PEG-PLA-NP $(2 \mu \mathrm{g} / \mathrm{mL})$ under different AMF treatment time, the magnetothermal conversion efficiency of tumor-bearing mice was detected by near-infrared thermal imaging camera and the tumor temperature curve was drawn. The antitumor efficiencies of the different nanocarriers in HeLa subcutaneous tumor-bearing mice were studied. Subcutaneous tumor-bearing mice $(n=25)$ were randomly divided into 5 groups. When the tumor volume reached $100 \mathrm{~mm}^{3}$, different drugs were injected. The experimental groups were DOX, DOX-Fe ${ }_{3} \mathrm{O}_{4}$-PEG-PLA-NPs, DOX-Fe $\mathrm{O}_{4}$-PEG-PLA-NPs (AMF), DOX-PEG-PLA-NPs (AMF) (DOX concentration of $10 \mathrm{mg} / \mathrm{kg}$ ), and the control group was injected with normal saline. The drug was injected every three days for eight treatment cycles. Mouse weights and tumor volumes were measured every 3 days during treatment (on days 14, 16, 18, 20, 22, 24 and 26). One week after the last injection, the mice were imaged to observe the tumor killing effects of the different treatments. The tumor-bearing mice were euthanized, and specimen tissues (heart, liver, spleen, lung, kidney and tumor) were taken for histological staining and sequencing. Its tumor volume was measured with calipers and calculated according to the formula: 


\section{Tumor volume $\left(\mathrm{cm}^{3}\right)=1 / 2$ (tumor length $\times$ tumor width2)}

(3)

Abdominal cavity tumor-bearing mouse model were randomly divided into five groups ( $n=5$ each group): (I) saline, (II) DOX, (III) DOX-PEG-PLA-NPs(AMF), (IV) DOX-Fe $\mathrm{O}_{3} \mathrm{O}_{4}$-PEG-PLA-NPs and (V) DOX-Fe $\mathrm{O}_{3}$-PEGPLA-NPs (AMF). Each mouse in the different groups was given a different treatment as designed, its survival was tracked separately throughout the experiment, and then a survival curve was drawn.

\section{Histological examination}

Different groups of tumor-bearing mice were sacrificed by inhalation anesthesia with excessive ether. The tumor, heart, liver, spleen, lung, and kidney were immediately removed, weighed, fixed with $10 \%$ formalin, and then paraffin-embedded. Histological changes and cell apoptosis were observed by hematoxylin and eosin (H\&E) staining, TUNEL staining and Ki67 immunohistochemical staining. The distribution of ferric oxide in different tissues was observed by Prussian blue staining. These images were obtained at $400 x$ magnification.

\section{Sequencing}

Tumor-bearing mice in different groups were sacrificed, and the tumor tissues were harvested for secondgeneration sequencing and proteomic detection.

\section{T2-weighted magnetic resonance imaging}

The T2-corrected MRI formaldehyde molecular probe DOX-Fe $\mathrm{O}_{4}$-PEG-PLA-NP relaxation rate was determined with different concentrations $(0.1,0.5,2.0,10.0,50.0$ and $100.0 \mu \mathrm{g} / \mathrm{mL}$ iron oxide concentration) of DOX-Fe ${ }_{3} \mathrm{O}_{4}$-PEG-PLA-NPs, USPIO standard product and $2 \mathrm{~mL}$ of glucosamine solution. Using a CE3.0T MR scanner (Shanghai Niumag Company, Shanghai, China), the above three different sets of solutions were placed on the 8-channel head coil, and the T2 relaxation rate data collected by the spin echo sequence (SE): TR 3000 msec; TE 10, 20, 40, 60, 80 and 100 msec was used. T2 Map was used to measure the $T_{2}$ values corresponding to different solution concentrations and the concentrationrelaxation rate curve was fit according to $1 / T_{2}$ and the solution concentration. The slope of the curve is the relaxation rate $\mathrm{r} 2$. A subcutaneous tumor-bearing mouse model was then established. When the mouse tumor diameter was greater than $1 \mathrm{~cm}$ for MRI scanning, an 8-channel mouse special MR coil was used to collect aberration parameters at position $T_{2}$. The instrument parameters were set as follows: TE $13.4 \mathrm{~mm}$, slice thickness $3 \mathrm{~mm}$, TR $660 \mathrm{msec}$, FOV 10, matrix: 512×512 $\mu \mathrm{m}$, NEX1. The mice were anesthetized by intraperitoneal injection of chloral hydrate (3\%), and MRI of the neck and chest was performed. First, a plain MRI scan was performed, and then DOX-Fe $\mathrm{O}_{3} \mathrm{O}_{4}$-PEG-PLA-NP solutions of different concentrations $(10.0,15.0,20.0,25.0,30.0 \mu \mathrm{g} / \mathrm{mL})$ were injected through the tail veins of the mice, with diatrizoate injection as a negative control. MRI scans were performed at different time points ( 5 minutes, 30 minutes, $1 \mathrm{~h}, 2 \mathrm{~h}, 4 \mathrm{~h}, 12 \mathrm{~h}$ and $24 \mathrm{~h}$ ) with the same sequence parameters as before. After scanning, the data before and after enhancement will be measured and analyzed. 


\section{Statistical analysis}

Statistical Package for the Social Sciences version 11.0 software (SPSS Inc., Chicago, IL, USA) was used for statistical analyses. The data are presented as the mean \pm standard deviation and were analyzed by one-way analysis of variance followed by Student's t-test. Statistical significance was set at alpha values of $p<0.001$ (very significant), $p<0.01$ (highly significant), and $p<0.05$ (significant).

\section{Abbreviations}

PEG: Polyethylene glycol; PLA: Polylactic acid; DOX: Doxorubicin; SPIONs: Superparamagnetic iron oxide nanoparticles; NP: Nanoparticle; DMSO: Dimethyl sulfoxide; EDTA: Ethylene diamine Tetraacetic Acid; AUC: Area under the curve; DLS: Dynamic light scatting; TEM: Transmission electron microscopy; ZP: Zeta potential; HNMR: Nuclear magnetic resonance spectroscopy; FBS: Fetal bovine serum; PBS:

Phosphate-buffered saline; HPLC: High performance liquid chromatography; MTT:(3-[4,5-dimethylthiazol2-yl]-2,5-diphenyltetrazolium bromide; RES: Reticuloendothelial system; EPR: Enhanced permeation and retention effect; MRI: Magnetic resonance imaging; AMF: Alternating magnetic field

\section{Declarations}

\section{Ethics approval and consent to participate}

The animal study protocols were performed in accordance with the Guide for the Care and Use of Laboratory Animals stipulated by the National Academy of Sciences and the National Institutes of Health (NIH publication 86-23, revised 1985) and approved by the Animal Care and Use Committee of Shandong University, Qilu Hospital (DWLL-2019-005) , Jinan, China.

\section{Consent for publication}

The authors declare that they agree to submit the article for publication.

\section{Availability of data and material}

All data are available in the main text or in the supplementary materials.

\section{Competing interests}

The authors declare that they have no competing interests. We confirm that this manuscript has been read and approved by all named authors and that there are no other persons who satisfied the criteria for authorship that are not listed in the manuscript. 


\section{Funding}

We acknowledge financial support from the National Natural Science Foundation of China (No. 81602286 to K. Song), the Taishan Scholar Project of Shandong Province (No. tsqn201812130 to K. Song).

\section{Authors' contributions}

$\mathrm{HL}$ and ZJL conceived and supervised the nanomaterials. CL and ZYW were responsible for the preparation and characterization of nanomaterials. YS and LL performed in vitro and in vivo experiments. $\mathrm{RC}$ performed magnetic resonance imaging. JYM was in charge of tissue distribution and pharmacokinetic experiments. XYP performed the antitumor efficacy of nanomaterials in vitro and in vivo. $X Q$ and $X T S$ analyzed all the experimental results. YS and LL edited the manuscript. $K S$ and $B H K$ conceived and supervised the study. All authors discussed the results and commented on the manuscript.

\section{Acknowledgments}

We thank the Translational Medicine Core Facility of Shandong University for consultation and the instrument availability that supported this work. We appreciate the Gynecological Oncology Laboratory of Qilu Hospital of Shandong University for supporting the cell-related experiments. Special thanks to Prof. Zaijun Lu and Dr. Kai Wang for their support with the experiments.

\section{References}

1. Arbyn M, Weiderpass E, Bruni L, De Sanjosé S, Saraiya M, Ferlay J, et al. Estimates of incidence and mortality of cervical cancer in 2018: a worldwide analysis. The Lancet Global Health. 2020;8:191203.

2. Liu B, Han L, Liu J, Han S, Chen Z, Jiang L. Co-delivery of paclitaxel and TOS-cisplatin via TATtargeted solid lipid nanoparticles with synergistic antitumor activity against cervical cancer. Int $\mathrm{J}$ Nanomedicine. 2017;12:955-68.

3. Fang J, Liu Y, Chen Y, Ouyang D, Yang G, Yu T. Graphene quantum dots-gated hollow mesoporous carbon nanoplatform for targeting drug delivery and synergistic chemo-photothermal therapy. Int $J$ Nanomedicine. 2018;13:5991-6007.

4. Dunne LJ, Manos G. Anthracyclines in the treatment of cancer. An overview. Virchows Archiv A. 1988;413:223-26.

5. Abbasi MM, Monfaredan A, Hamishehkar H, Jahanban-Esfahlan R. New formulated "DOX-MTXloaded nanoparticles" down- regulate HER2 gene expression and improve the clinical outcome in OSCCs model in rat: the effect of IV and oral modalities. Asian Pac J Cancer Prev. 2014;15:9355-60. 
6. Xia Y, Xu T, Zhao M, Hua L, Chen Y, Wang C, et al. Delivery of Doxorubicin for Human Cervical Carcinoma Targeting Therapy by Folic Acid-Modified Selenium Nanoparticles. Int J Mol Sci. 2018;19 (11).

7. Ordikhani F, Erdem AM, Marcelo R, Sahin I, Grigsby P, Schwarz J, et al. Drug Delivery Approaches for the Treatment of Cervical Cancer. Pharmaceutics. 2016;8:23.

8. Zheng Y, Chen H, Zeng X, Liu Z. Surface modification of TPGS-b-(PCL-ran-PGA) nanoparticles with polyethyleneimine as a co-delivery system of TRAIL and endostatin for cervical cancer gene therapy. Nanoscale Res Lett. 2013;8:161.

9. Peng XH, Qian X, Hui M, Wang AY, Dong MS. Targeted magnetic iron oxide nanoparticles for tumor imaging and therapy. Int J Nanomed. 2008;3:311-21.

10. Arami $\mathrm{H}$, Khandhar A, Liggitt $\mathrm{D}$, Krishnan KM. In vivo delivery, pharmacokinetics, biodistribution and toxicity of iron oxide nanoparticles. Chem Soc Rev. 2015;44:8576-607.

11. Bano S, Nazir S, Nazir A, Munir S, Mahmood T, Afzal M, et al. Microwave-assisted green synthesis of superparamagnetic nanoparticles using fruit peel extracts: surface engineering, T 2 relaxometry, and photodynamic treatment potential. Int J Nanomedicine. 2016;11:3833-48.

12. Anselmo AC, Mitragotri S. A Review of Clinical Translation of Inorganic Nanoparticles. AAPS J. 2015;17:1041-54.

13. Cai J, Miao YQ, Li L, Fan HM. Facile Preparation of Gold-Decorated Fe(3)O(4) Nanoparticles for CT and MR Dual-Modal Imaging. Int J Mol Sci. 2018;19.

14. Gu L, Fang RH, Sailor MJ, Park JH. In Vivo Clearance and Toxicity of Monodisperse Iron Oxide Nanocrystals. ACS Nano. 2012;6:4947-54.

15. Kumar P, Agnihotri S, Roy I. Preparation and characterization of superparamagnetic iron oxide nanoparticles for magnetically guided drug delivery. Int J Nanomedicine. 2018;13:43-6.

16. Alphandéry E. Biodistribution and targeting properties of iron oxide nanoparticles for treatments of cancer and iron anemia disease. Nanotoxicology. 2019;13:573-96.

17. Jia YH, Yuan M, Yuan HD, Huang XL, Sui X, Cui XM, et al. Co-encapsulation of magnetic Fe3O4 nanoparticles and doxorubicin into biodegradable PLGA nanocarriers for intratumoral drug delivery. Int J Nanomed. 2012;7:1697-708.

18. Gobbo OL, Sjaastad K, Radomski MW, Volkov Y, Prina-Mello A. Magnetic Nanoparticles in Cancer Theranostics. Theranostics. 2015;5:1249-63.

19. King MA, Clanton TL, Laitano O. Hyperthermia, dehydration, and osmotic stress: unconventional sources of exercise-induced reactive oxygen species. Am J Physiol Regul Integr Comp Physiol. 2016;310:105-14.

20. Lee BK, Yun Y, Park K. PLA micro- and nano-particles. Adv Drug Deliv Rev. 2016;107:176-91.

21. Wang Y, Zhang S, Benoit DSW. Degradable poly(ethylene glycol) (PEG)-based hydrogels for spatiotemporal control of siRNA/nanoparticle delivery. J Control Release. 2018;287:58-66. 
22. Li Y, Yang J, Xu B, Gao F, Wang W, Liu W. Enhanced Therapeutic siRNA to Tumor Cells by a pHSensitive Agmatine-Chitosan Bioconjugate. ACS Appl Mater Interfaces. 2015;7:8114-24.

23. Ouahab A, Cheraga N, Onoja V, Shen Y, Tu J. Novel pH-sensitive charge-reversal cell penetrating peptide conjugated PEG-PLA micelles for docetaxel delivery: in vitro study. Int J Pharm. 2014;466:233-45.

24. Xu CF, Zhang HB, Sun CY, Liu Y, Shen S, Yang XZ, et al. Tumor acidity-sensitive linkage-bridged block copolymer for therapeutic siRNA delivery. Biomaterials. 2016;88:48-59.

25. Nurcahyanti AD, Wink M. L-Canavanine potentiates the cytotoxicity of doxorubicin and cisplatin in arginine deprived human cancer cells. PeerJ. 2016;4:e1542.

26. Lim C, Youn YS, Lee KS, Hoang NH, Sim T, Lee ES, et al. Development of a robust pH-sensitive polyelectrolyte ionomer complex for anticancer nanocarriers. Int J Nanomedicine. 2016;11:703-13.

27. Golombek SK, May JN, Theek B, Appold L, Drude N, Kiessling F, et al. Tumor targeting via EPR: Strategies to enhance patient responses. Adv Drug Deliv Rev. 2018;130:17-38.

28. Spicer CD, Jumeaux C, Gupta B, Stevens MM. Peptide and protein nanoparticle conjugates: versatile platforms for biomedical applications. Chem Soc Rev. 2018;47:3574-620.

29. Schleich N, Po C, Jacobs D, Ucakar B, Gallez B, Danhier F, et al. Comparison of active, passive and magnetic targeting to tumors of multifunctional paclitaxel/SPIO-loaded nanoparticles for tumor imaging and therapy. J Control Release. 2014;194:82-91.

30. Bhattacharyya S, Kudgus RA, Bhattacharya R, Mukherjee P. Inorganic nanoparticles in cancer therapy. Pharm Res. 2011;28:237-59.

31. Westermann AM, Jones EL, Schem BC, van der Steen-Banasik EM, Koper P, et al. First results of triplemodality treatment combining radiotherapy, chemotherapy, and hyperthermia for the treatment of patients with stage IIB, III, and IVA cervical carcinoma. Cancer. 2005;104:763-70.

32. Ghazanfari MR, Kashefi M, Shams SF, Jaafari MR. Perspective of Fe304Nanoparticles Role in Biomedical Applications. Biochemistry Research International. 2016; 2016:1-32.

33. Kievit FM, Wang FY, Fang C, Mok H, Wang K. Silber JR, et al. Doxorubicin loaded iron oxide nanoparticles overcome multidrug resistance in cancer in vitro. J Control Release. 2011;152(1):7683.

34. Chen L, Xie J, Wu H, Zang F, Ma M, Hua Z, et al. Improving sensitivity of magnetic resonance imaging by using a dual-targeted magnetic iron oxide nanoprobe. Colloids and surfaces. B, Biointerfaces. 2018; 161:339-46.

35. Du Y, Lai PT, Leung CH, Pong PW. Design of superparamagnetic nanoparticles for magnetic particle imaging (MPI). Int J Mol Sci. 2013;14:18682-710.

36. Kizilel S, Ergenc TI, Acar H, Yar Y. RGDS-functionalized polyethylene glycol hydrogel-coated magnetic iron oxide nanoparticles enhance specific intracellular uptake by HeLa cells. Int J Nanomed. 2012;7:1903-20. 
a<smiles>CC(C)OC(C)C(=O)C(C)C</smiles>

PLA<smiles>CCC(C)(O)CCOC(C)C</smiles>

b

PEG

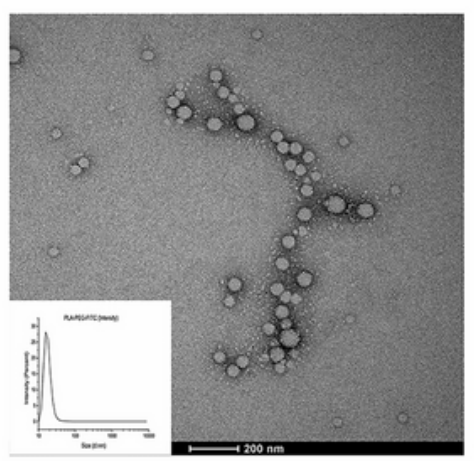

C

d

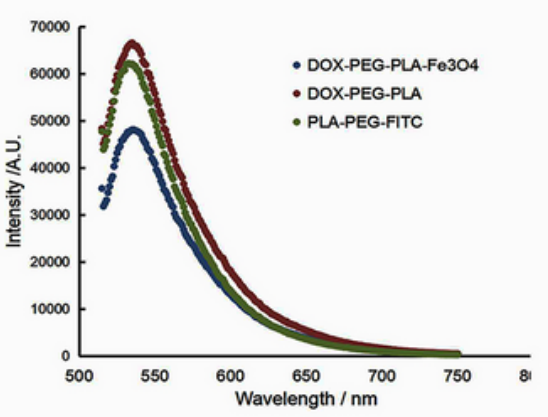

PEG-PLA
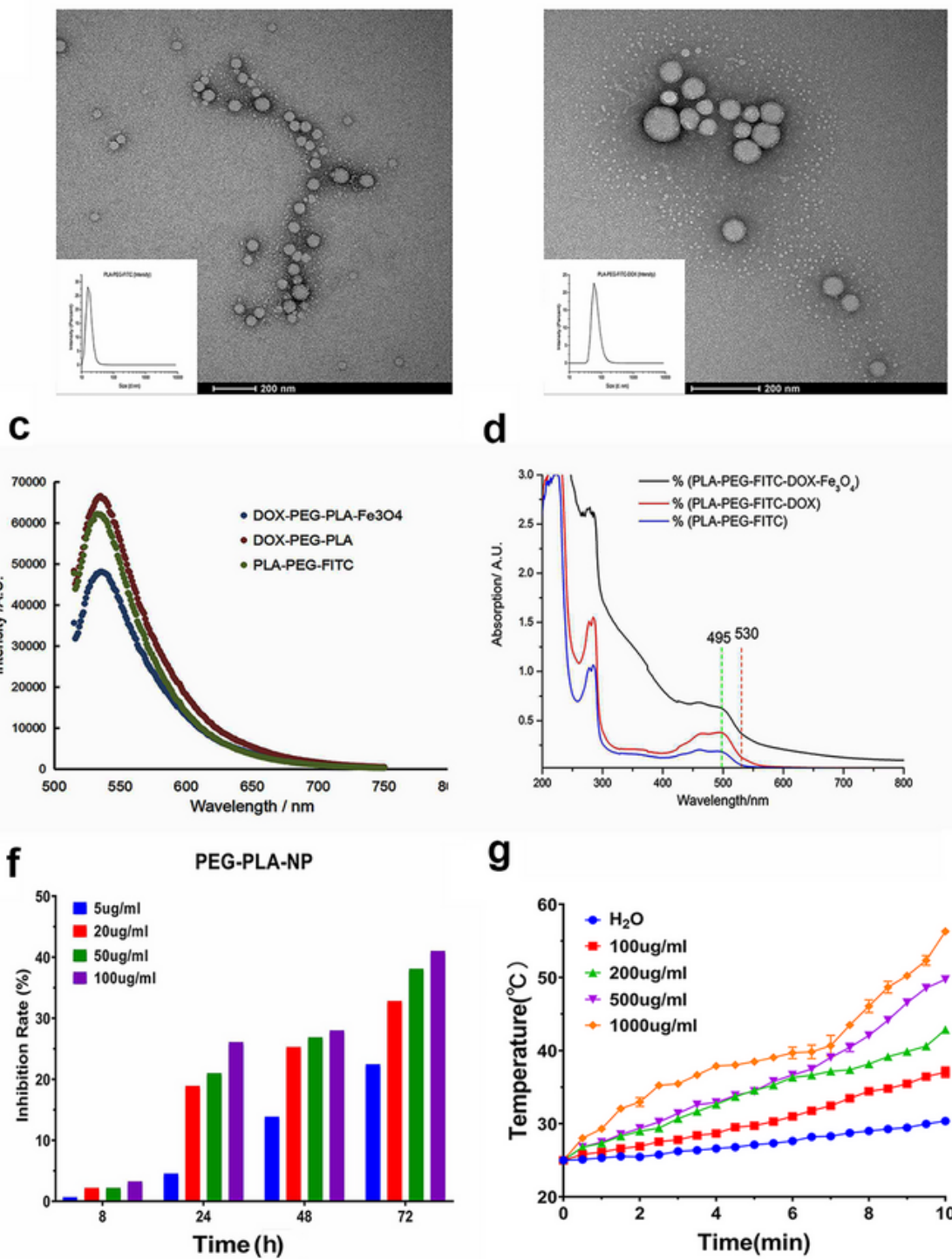

g
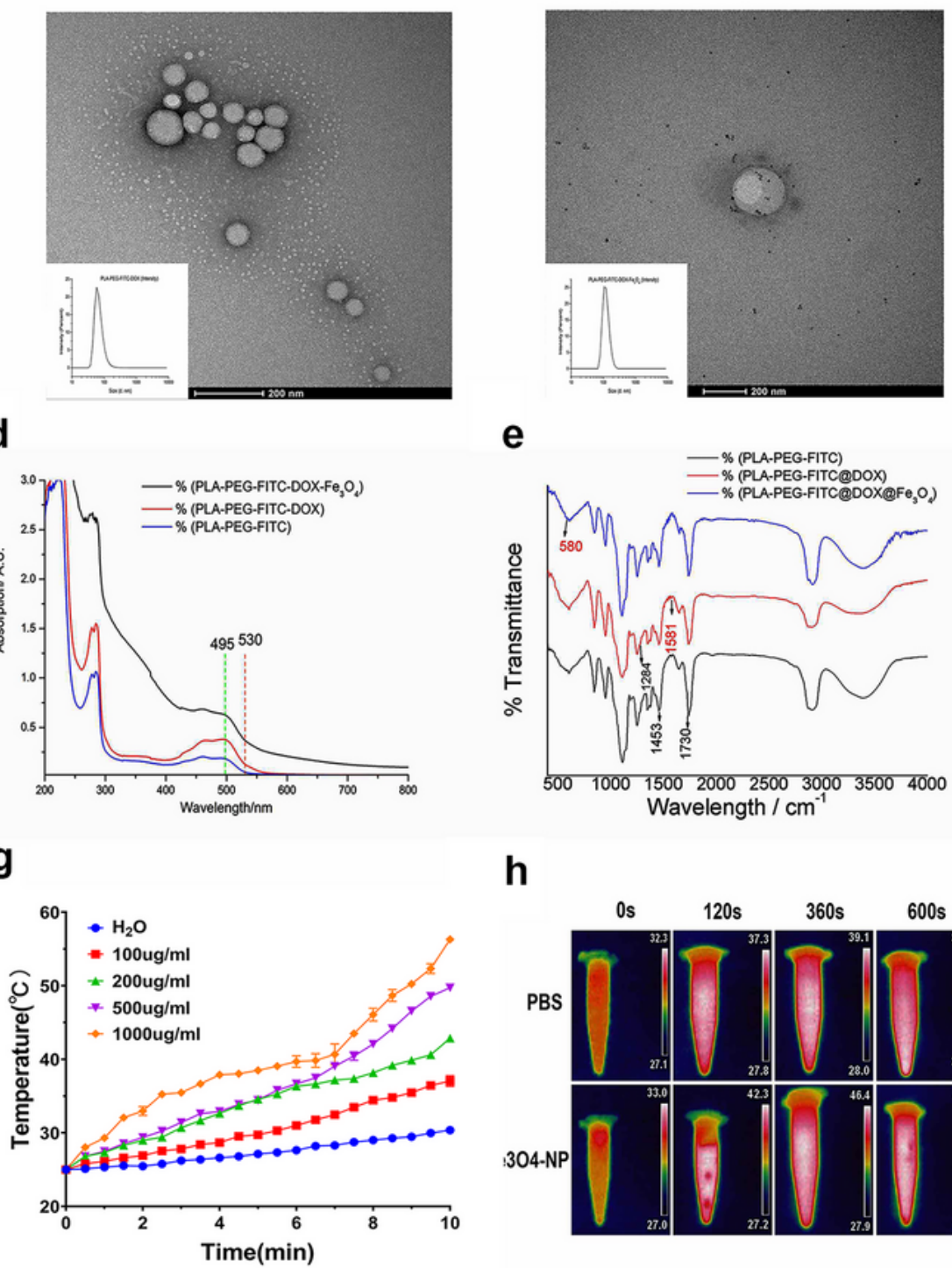

e

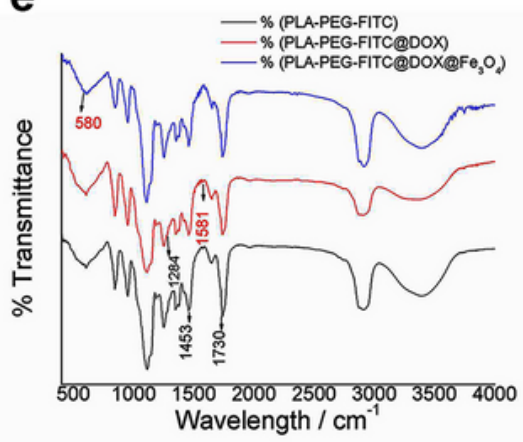

h

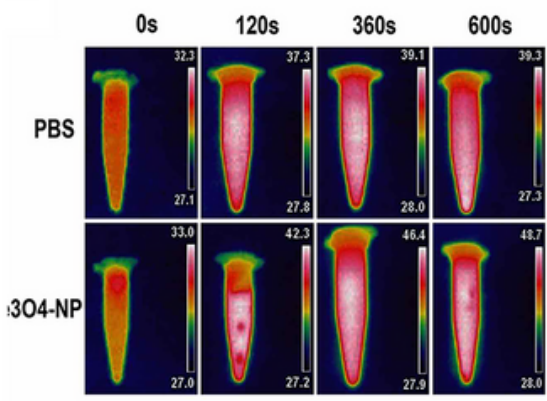

Figure 1

Characterization of DOX-Fe304-PEG-PLA-NPs. (a) Flow chart of DOX-Fe304-PEG-PLA. (b) TEM images and the diameter of PEG-PLA-NP, DOX-PEG-PLA-NP and DOX-Fe304-PEG-PLA-NP measured using DLS. (c) Fluorescence spectrum of FITC coupled with different nanocarriers. (d) UV absorption spectra of the 
PEG-PLA-FITC empty nanoparticles (blue), PEG-PLA-FITC nanoparticles containing DOX (red), and PEGPLA-FITC nanoparticles containing Fe304 and DOX (black). (e) Infrared spectra of PEG-PLA-FITC, DOXPEG-PLA-FITC and DOX-Fe304-PEG-PLA-FITC nanoparticles. (f) Toxicity of PEG-PLA nanomaterials using MTT. (g) Magneto-thermal transformation of different Fe304 concentrations $(100-1000 \mu \mathrm{g} / \mathrm{mL})$ under the alternating magnetic field. (h) Photothermal images of Fe304-DOX-PEG-PLA-FITC nanoparticles and water under the alternating magnetic field.
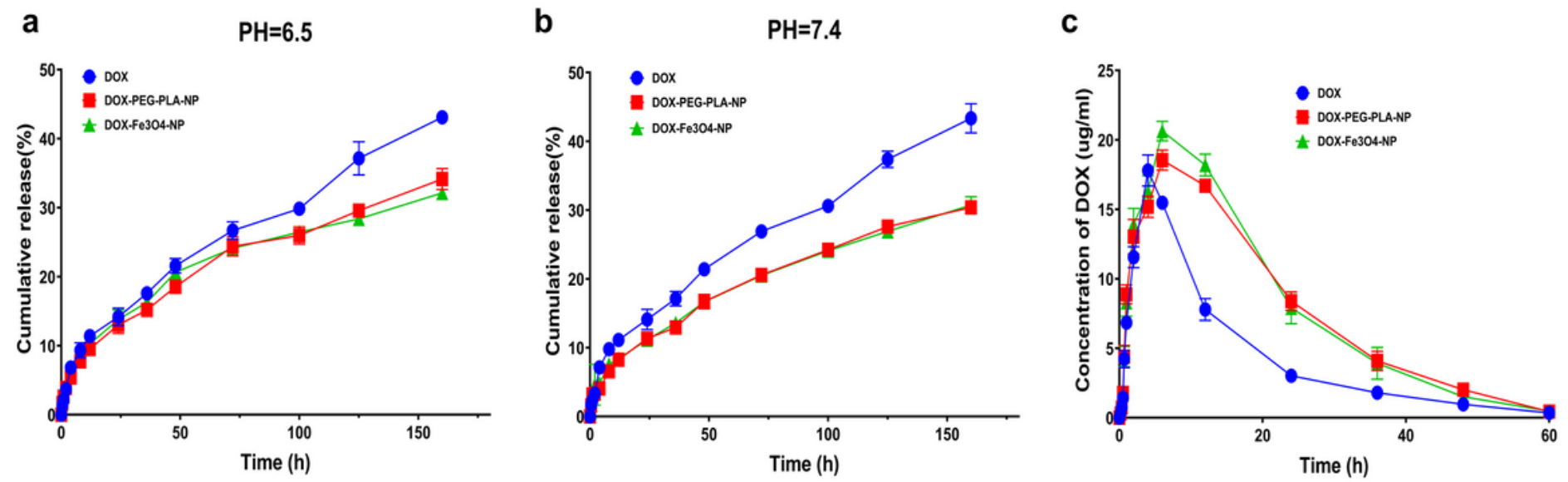

\section{Figure 2}

DOX and Fe304 could be released from the DOX-Fe304-PEG-PLA-FITC nanoparticles. (a) The cumulative release of DOX from the free DOX solution, DOX-PEG-PLA-NPs and DOX-Fe304-PEG-PLA-NPs at pH=6.5. (b) The cumulative release of DOX from the free DOX solution, DOX-PEG-PLA-NPs and DOX-Fe304-PEGPLA-NPs at $\mathrm{pH}=7.4$. (c) Pharmacokinetics of HeLa tumor-bearing mice after intravenous injection of DOX, DOX-PEG-PLA-NPs and DOX-Fe304-PEG-PLA-NPs. The free DOX group was used as a control. 
a

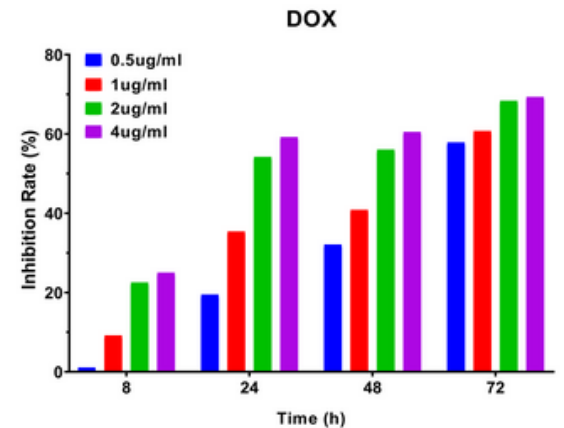

DOX(AMF)

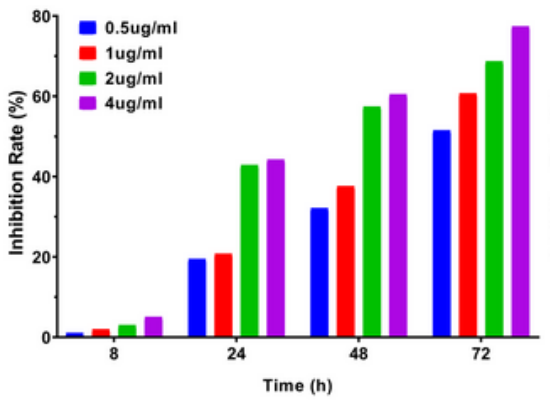

b

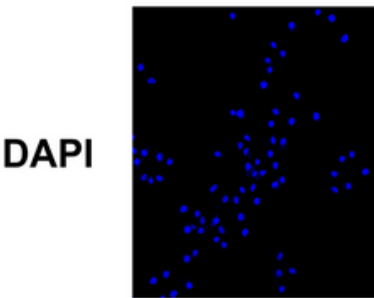

ROS

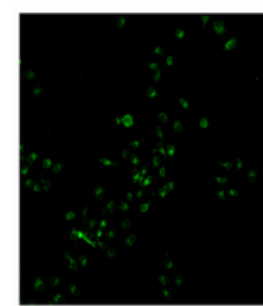

Merge

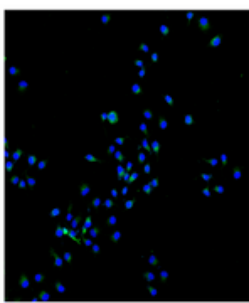

DOX-PEG-PLA-NP

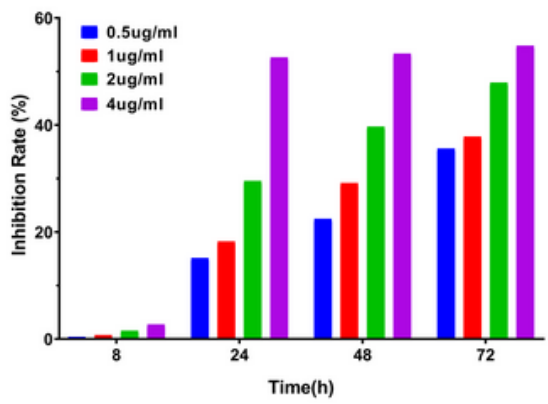

DOX-PEG-PLA-NP(AMF)
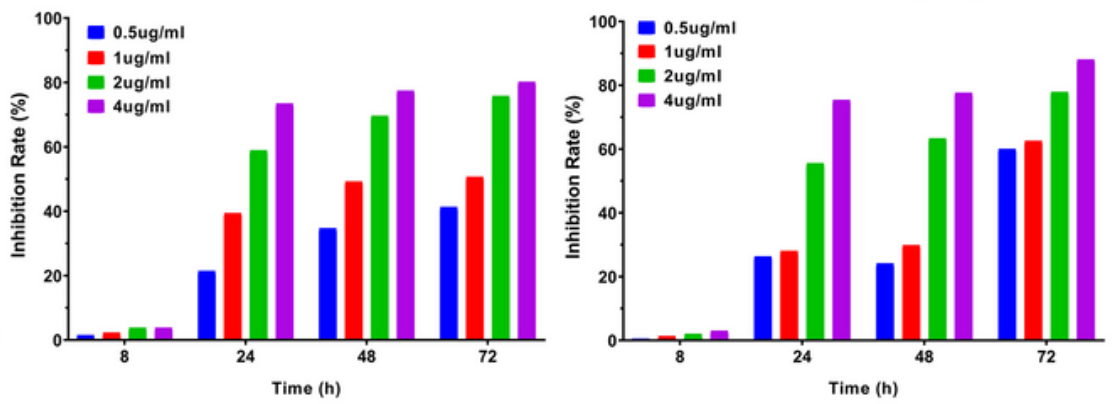

DOX-Fe304-NP

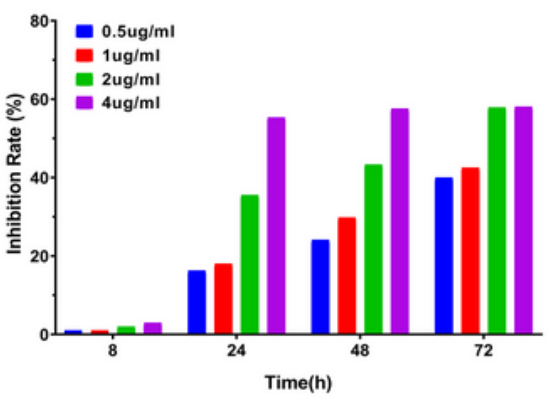

DOX-Fe304-NP(AMF)
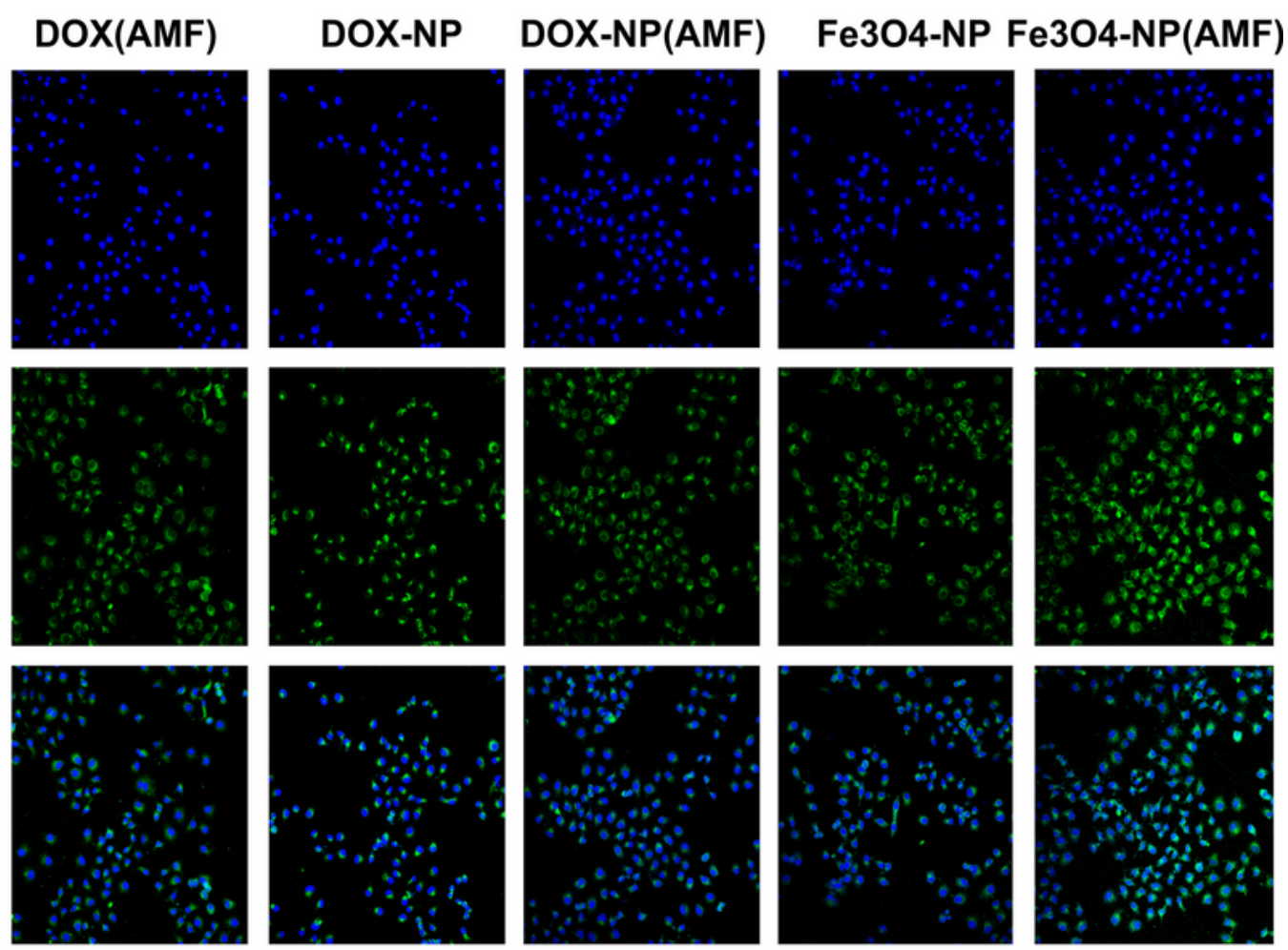

Figure 3

HeLa cell cytotoxicity of simple and drug-loaded nanomaterials in vitro. (a) HeLa cytotoxicity after treatment with free DOX, DOX-PEG-PA-NPs and DOX-Fe304-PEG-PLA-NPs with or without alternating magnetic field at different DOX concentrations $(0.5,1.0,2.0$ and $4.0 \mu \mathrm{g} / \mathrm{mL})$ for different time $(8 \mathrm{~h}, 24 \mathrm{~h}$, $48 \mathrm{~h}$ and $72 \mathrm{~h}$ ). (b) The ability of different groups to produce reactive oxygen species (ROS) with or 
without alternating magnetic field. Blue fluorescence represents DAPI staining nucleus, and green fluorescence represents ROS content.

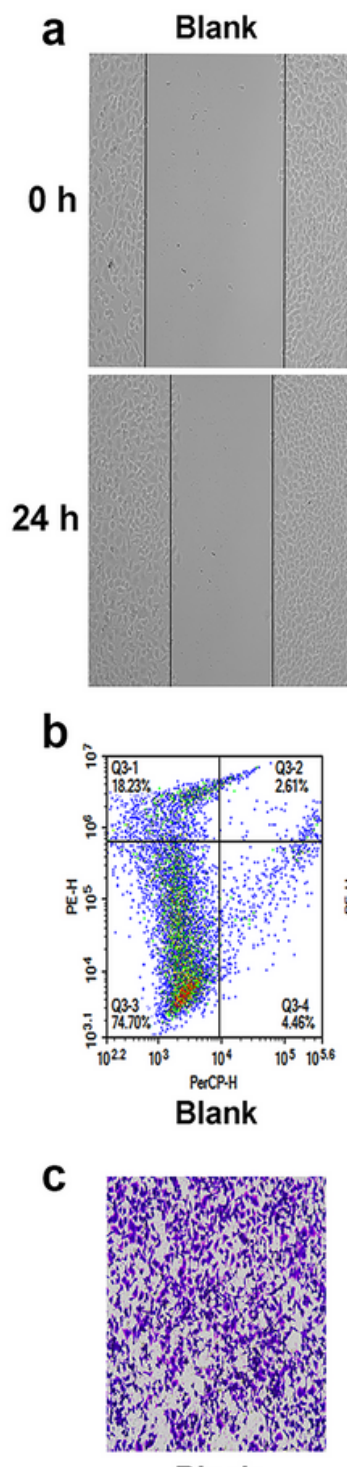

Blank

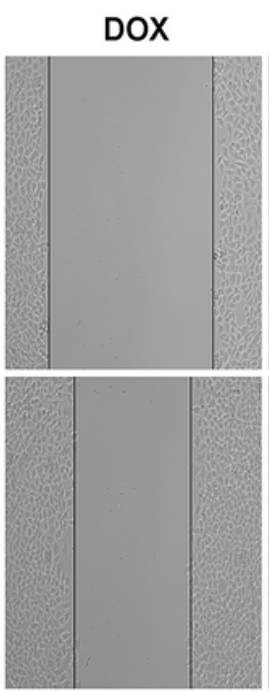

DOX-PEG-PLA
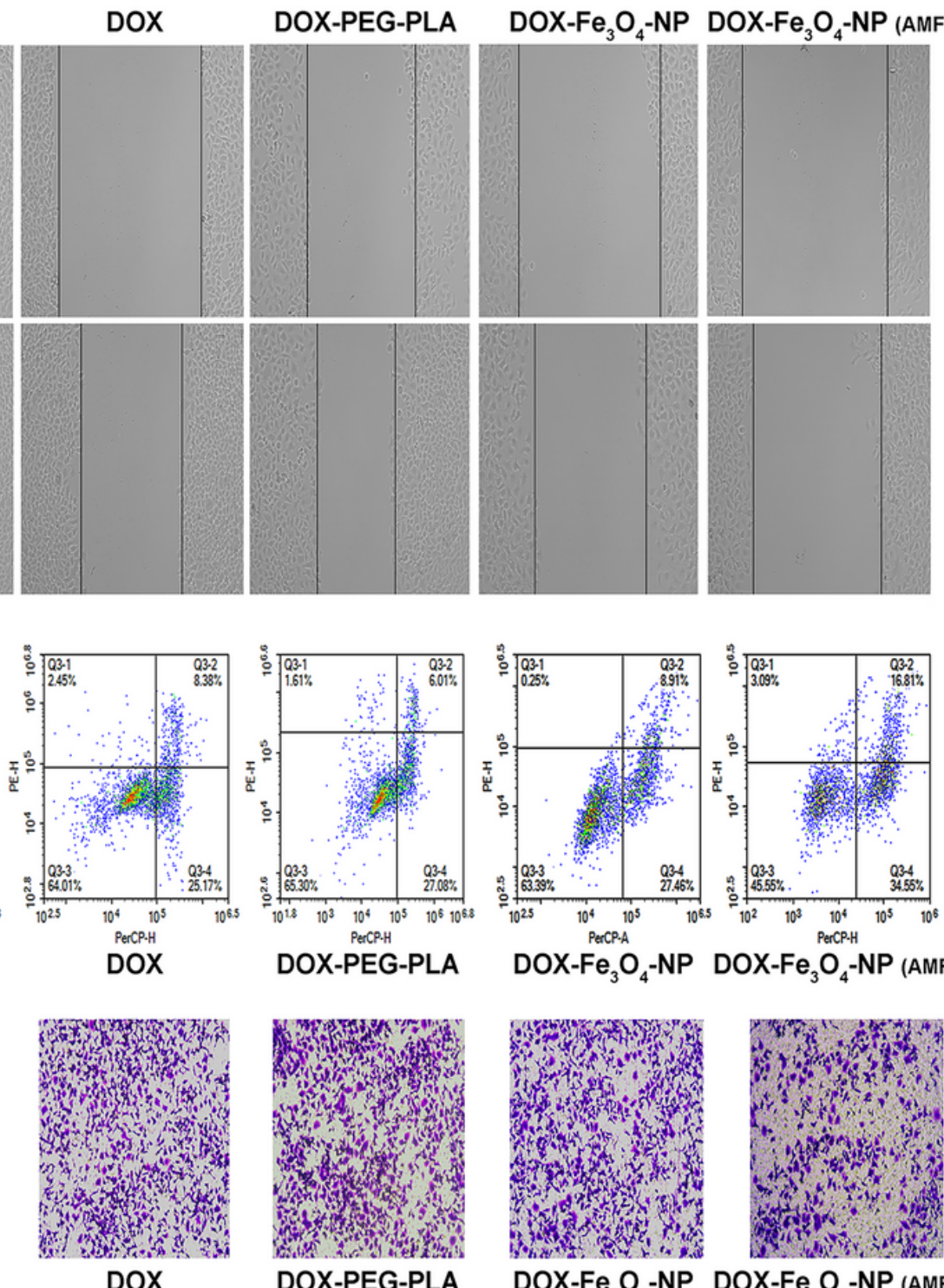

DOX
DOX-PEG-PLA

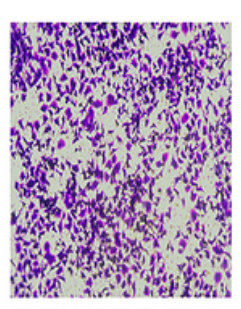

DOX-PEG-PLA
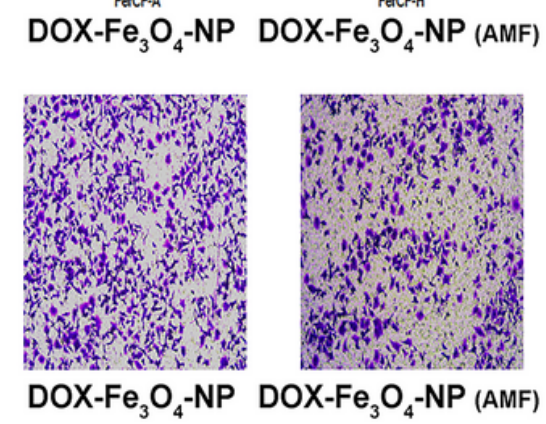

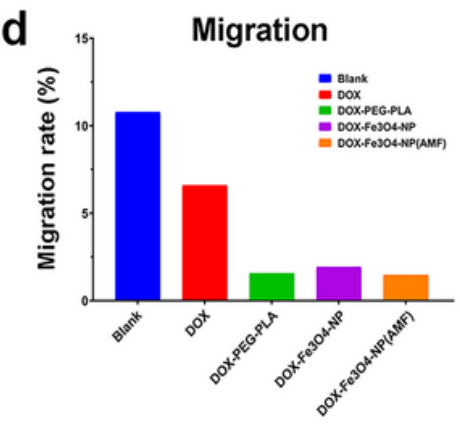

e
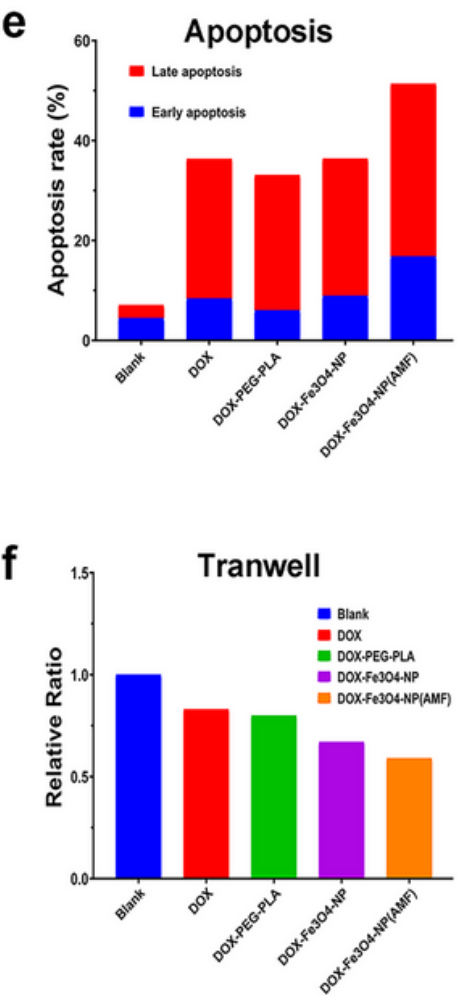

Figure 4

Effects of the different drug-loaded nanomaterials on the invasion and migration ability and apoptosis of HeLa cells in vitro. $(a, d)$ The migration ability of tumor cells in different groups was detected by a scratch test after $24 \mathrm{~h}$ of incubation. (b, e) Apoptosis detection via annexin V-FITC/PI assay in HeLa cells induced by DOX, DOX-PEG-PA-NPs DOX-Fe304-PEG-PLA-NP and DOX-Fe304-PEG-PLA-NP(AMF) after $24 \mathrm{~h}$ of incubation. PBS was used as a control. (c, f) Transwell assays were used to detect the influence of the different treatments on the invasion ability of tumor cells. 


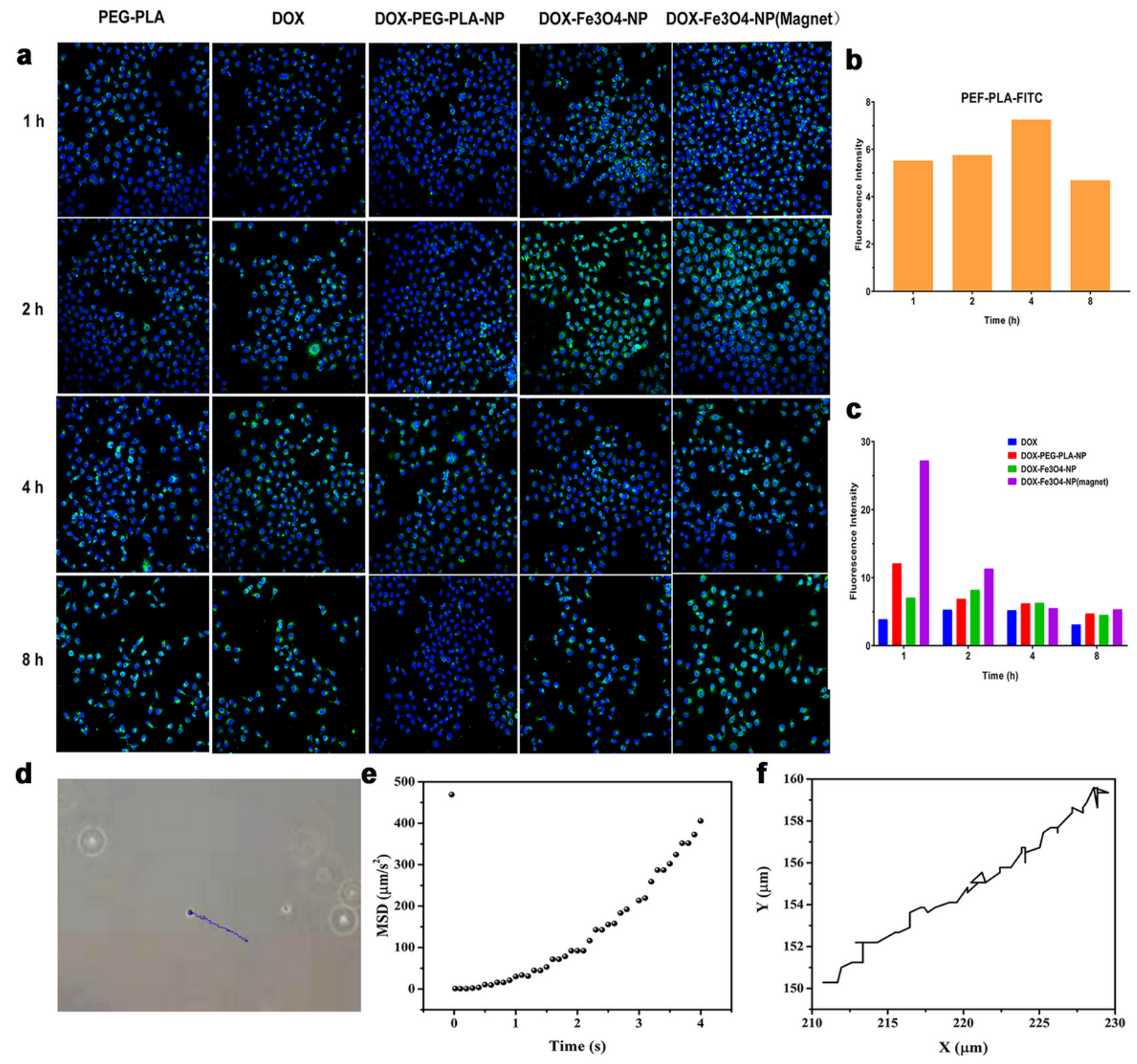

Figure 5

Cellular uptake of the nanomaterials in different groups were detected by fluorescence microscopy. (a) Uptake of PEG-PLA-NPs was observed by fluorescence microscopy after different incubation times. FITC on the surface of the PEG-PLA nanomaterials appears green under the excitation light, and DAPI was used to stain the nucleus. In the other drug groups, DOX showed green fluorescence after fluorescence excitation, and DAPI nuclear staining showed blue fluorescence. (b) Fluorescence intensity of the PEGPLA-NPs analyzed by Image J software after different incubation times. (c) The fluorescence intensities in the different DOX groups analyzed by Image $J$ software after different incubation times. (d) Time-lapse 
images depicting the movement of the nanoparticles toward Hela cells. (e-f) The mean square displacement diagrams and plane trajectories of nanoparticles under the attraction of an external magnet
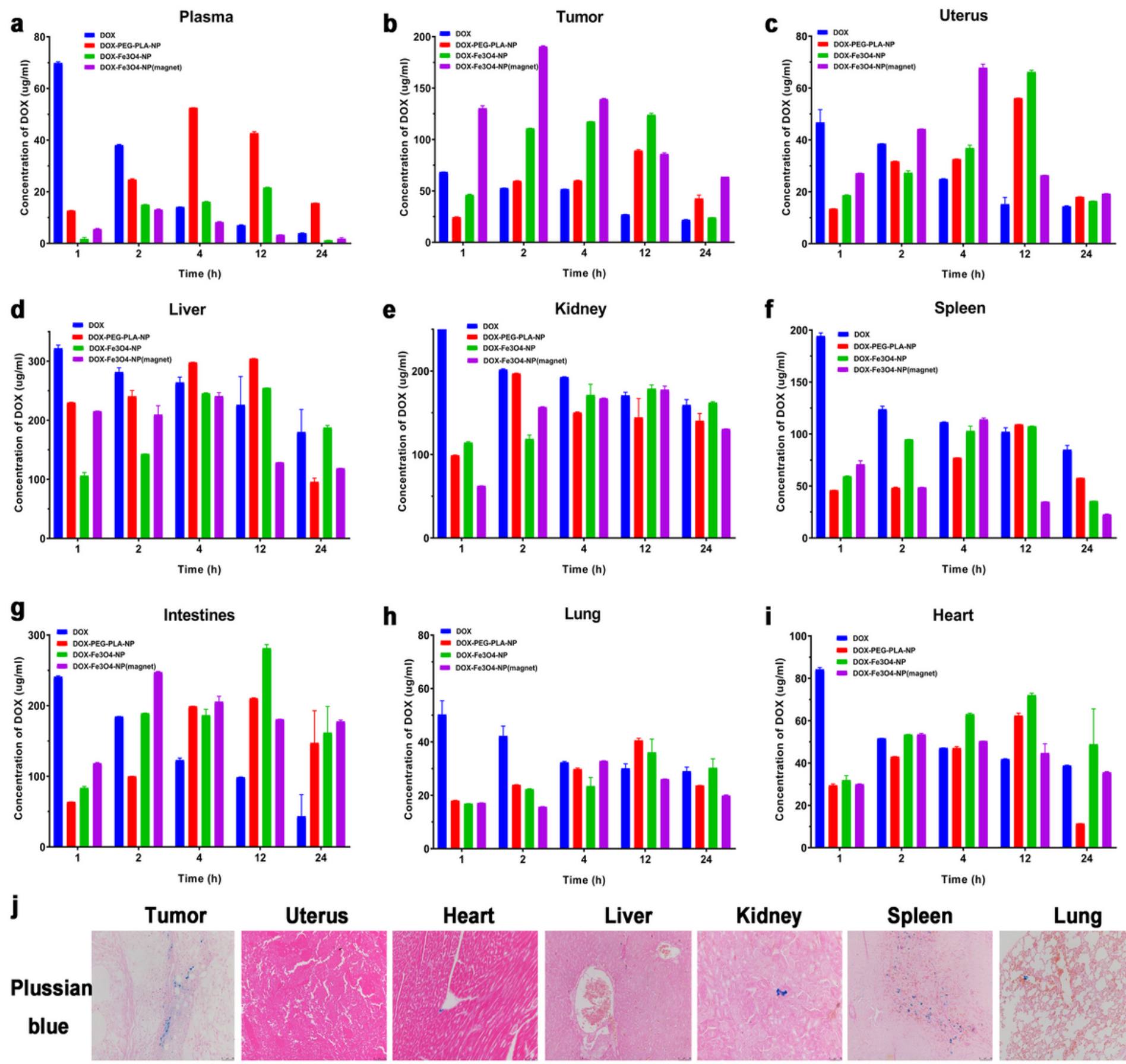

Heart

Liver

Kidney

Spleen

Lung

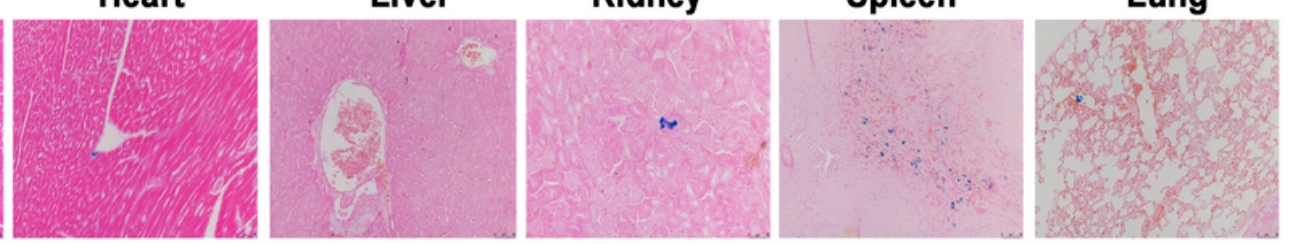

Figure 6

The biodistribution of DOX in different tissues at different time points in tumor-bearing mice treated. (a-i) Biodistribution of different tissue in tumor-bearing mice after intravenous injection of DOX, DOX-PEG-PANPs, DOX-Fe304-PEG-PLA-NPs and DOX-Fe304-PEG-PLA-NPs (magnet field) (DOX concentration of 10 $\mathrm{mg} / \mathrm{kg}$ ). (j) Prussia blue staining in tumor from mice with the DOX-Fe304-PEG-PLA-NP group treatments. 

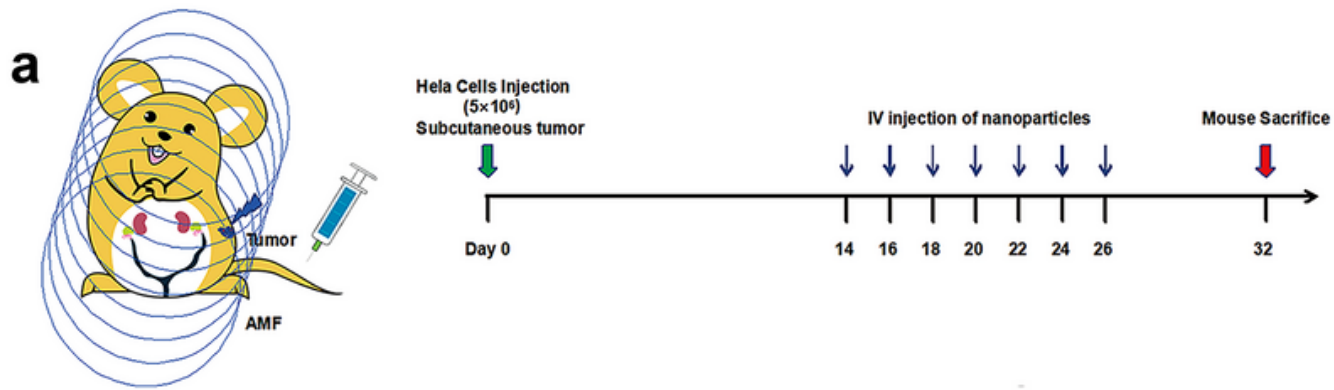

G1, Saline

G2, DOX-Fe304-PEG-PLA-NP G3, DOX

G4, DOX-PEG-PLA-NP(AMF)

G5, DOX-Fe304-PEG-PLA-NP(AMF)
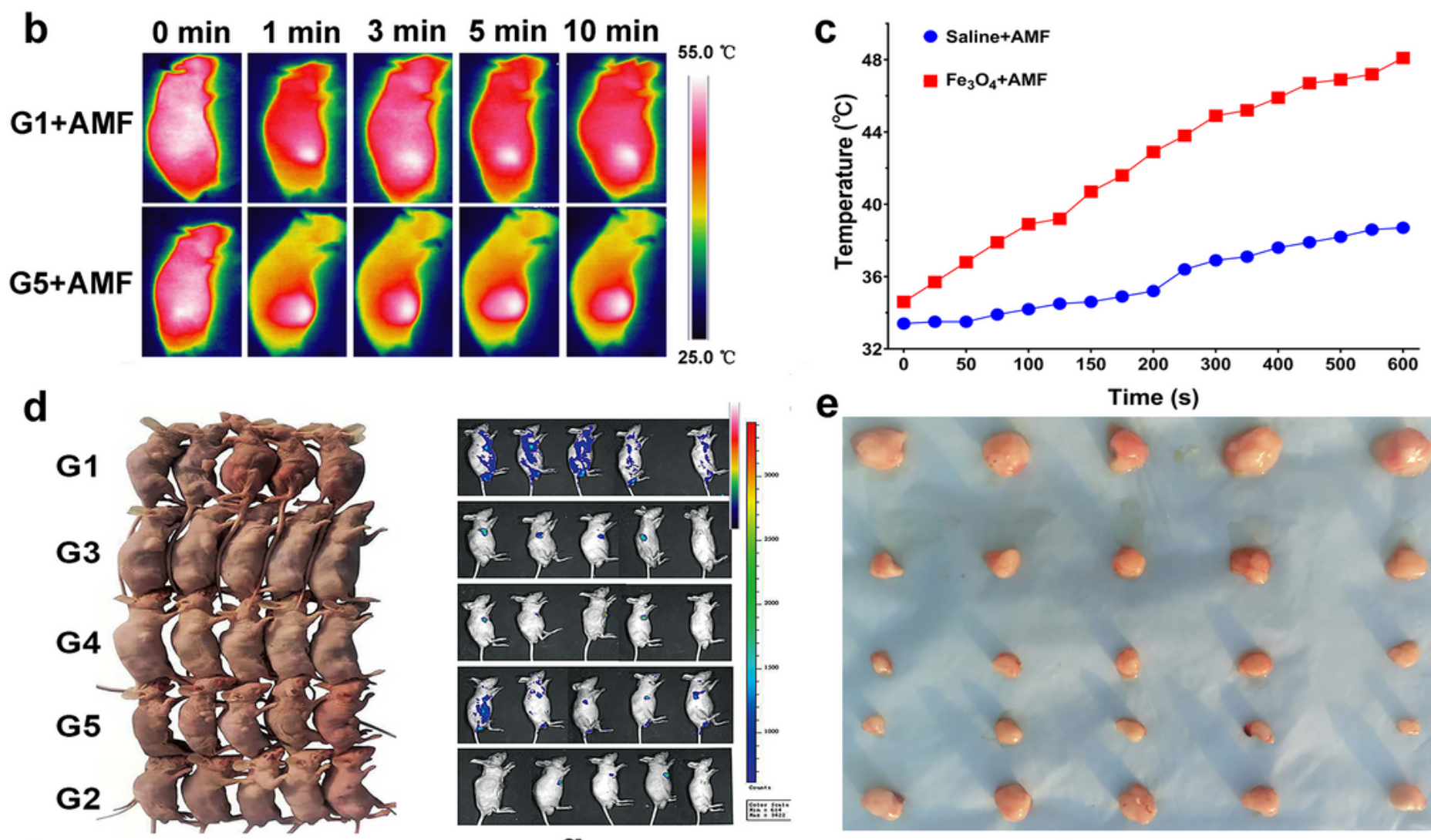

f

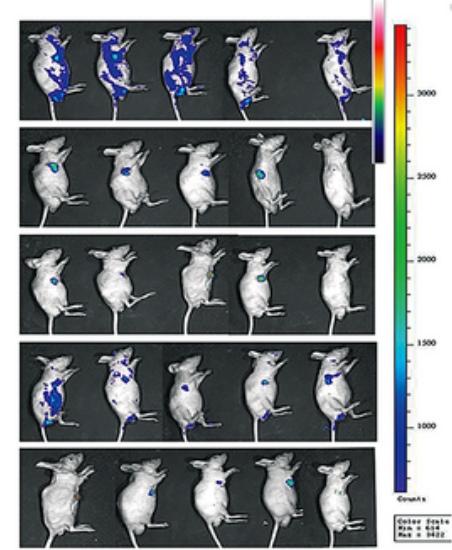

g
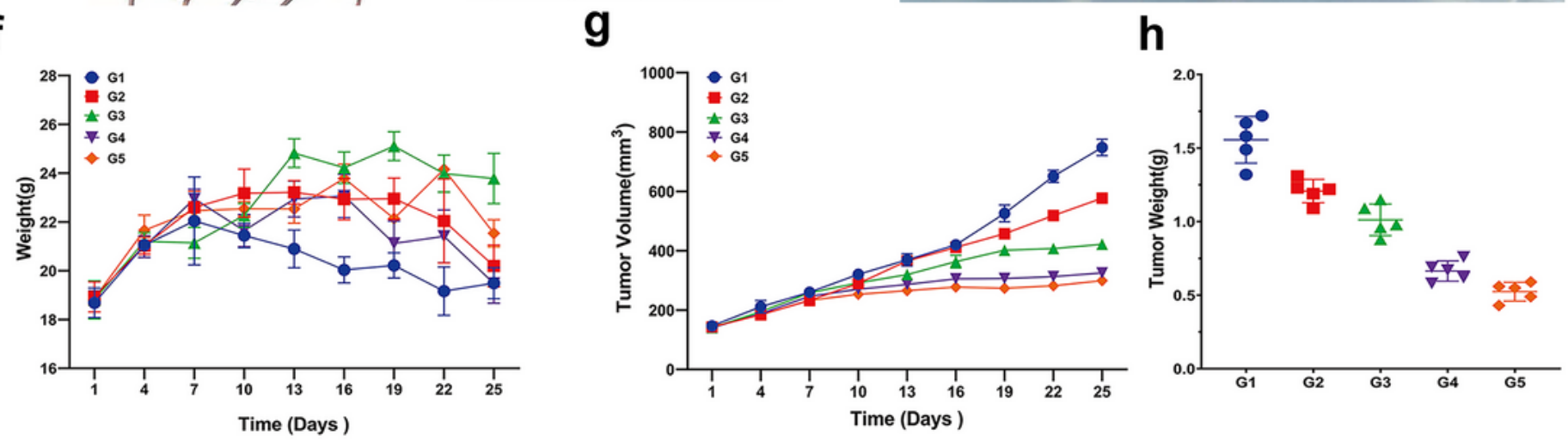

\section{Figure 7}

In vivo antitumor effects of different treatment groups. (a) Diagram of treatment plans in subcutaneous tumor-bearing mouse models with different treatments. (b) Image taken with IR thermal camera of Hela tumor-bearing mice with different treatments under AMF. (c) Tumor temperature curves of tumor region under AMF. (d-e) Fluorescence imaging and photographsmap of tumor tissue in tumor-bearing mice. (f) Relative mouse body weight during treatment. (g) Growth curve of the subcutaneous tumor-bearing 
mouse model with indicated treatments (mean \pm s.d., $n=5$ /group) (h) Weight of the primary tumor from the mouse after treatment. Data are shown as mean $\pm \operatorname{SD}(n=5)$.

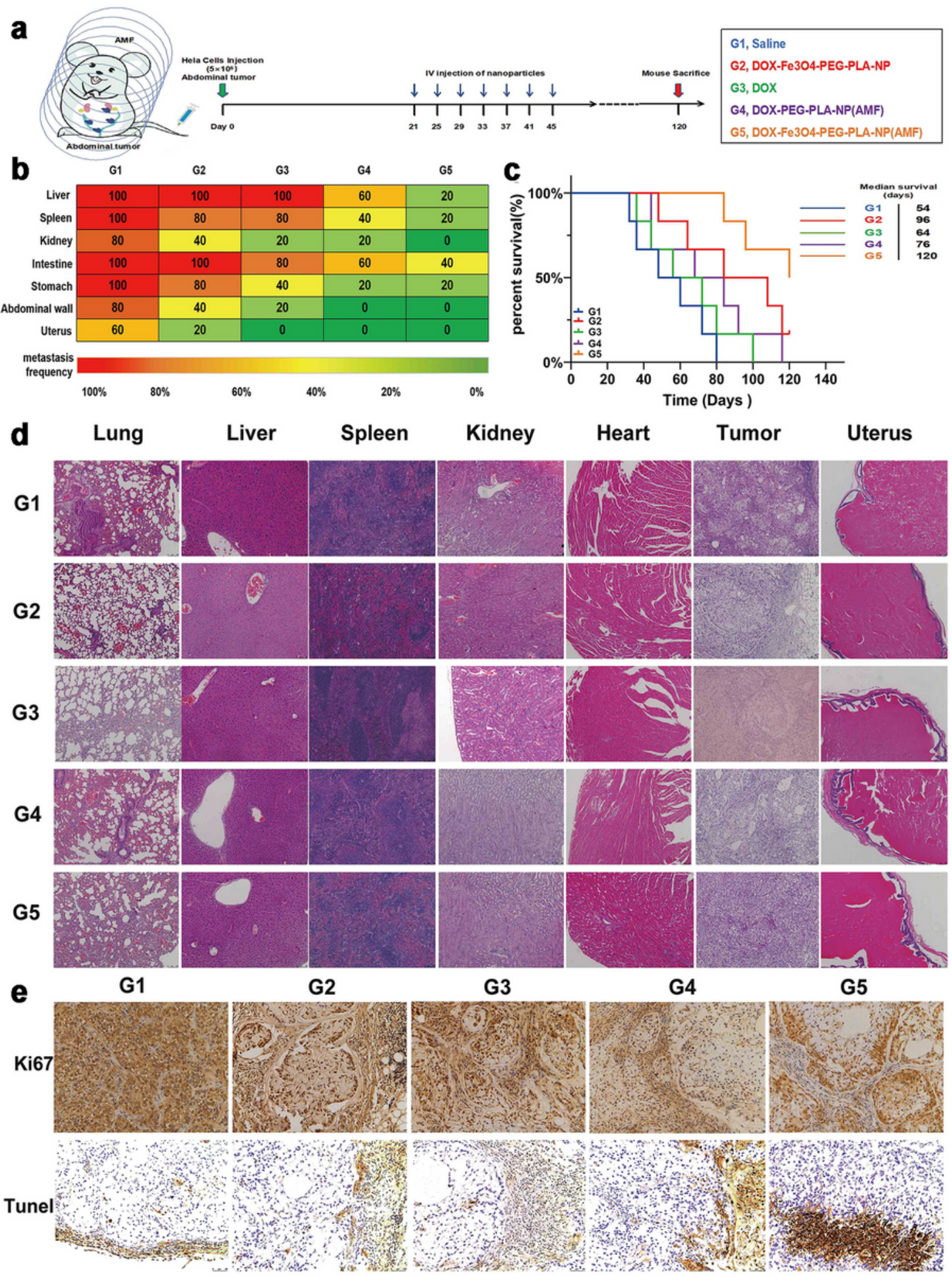

\section{Figure 8}

In vivo survival verification with indicated treatments. (a) Diagram of treatment plans in abdominal tumor-bearing mouse models with different treatments. (b) Heat map of tumor metastasis frequency in tissues and organs. (c) Kaplan-Meier survival graph of abdominal tumor-bearing mice after treatment. (d) 
H\&E analysis of different tissues/organs with different treatments. (e) Ki67 and TUNEL staining of tumor tissue with different treatments.
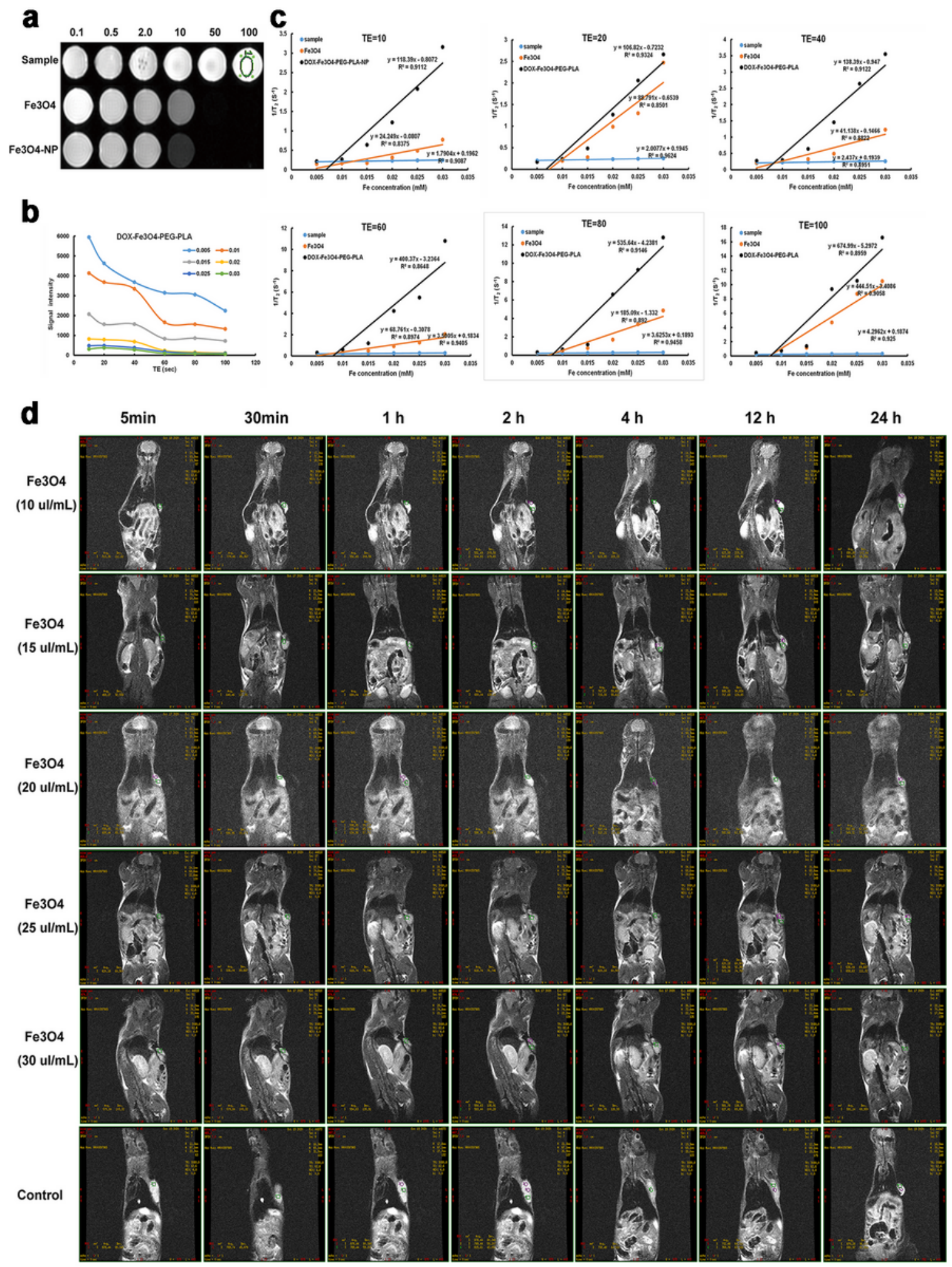

\section{Figure 9}

The sequencing results of the tumor tissues in the different groups. (a) The proteomics KEGG pathway database. (b) The sequencing KEGG pathway database. (c) The proteomics heatmap illustrating clustered matrix of sample-wise similarities based on whole tumor profiles of primary cervical tumors 
with different treatment. (d) The sequencing heatmap illustrating clustered matrix of sample-wise similarities based on whole tumor profiles of primary cervical tumors with different treatment.
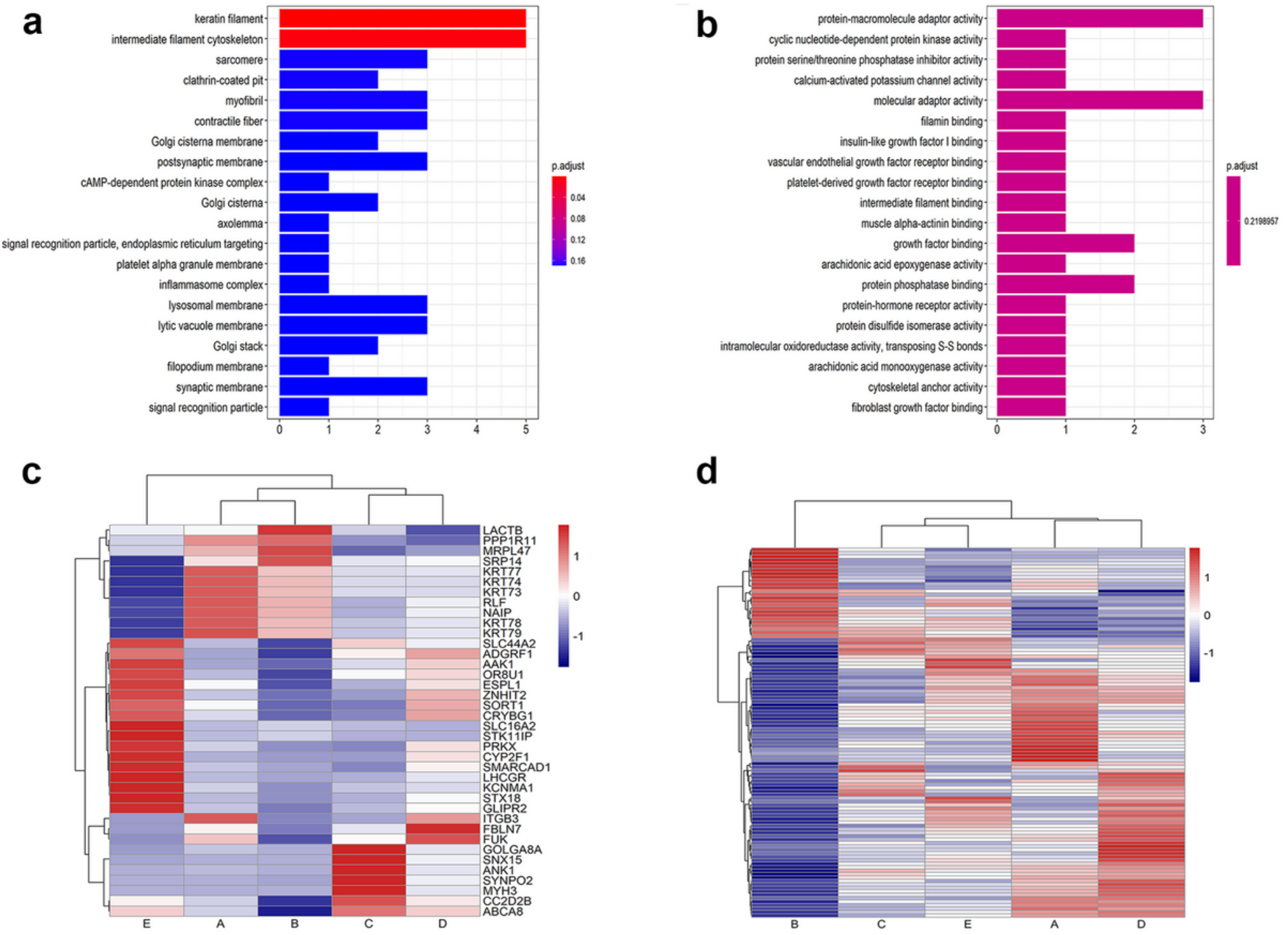

\section{Figure 10}

In vitro and in vivo MRI results of different materials. (a) MRI results of different materials under different TE and concentration conditions in vitro. (b) Changes in the signal strength of the DOX-Fe304-PEG-PLANPs under different TE conditions in vitro. (c) The relaxation rate of different materials under different TE $(10,20,40,60,80$ and 100) conditions. (d) MRI of mice injected intravenously with different materials at different times.

\section{Supplementary Files}

This is a list of supplementary files associated with this preprint. Click to download.

- GraphicalAbstarct.png

- figures1.tif 